\title{
Effective WIMPs
}

\author{
Spencer Chang ${ }^{a}$, Ralph Edezhath ${ }^{b}$, Jeffrey Hutchinson ${ }^{b}$, and Markus Luty ${ }^{b}$ \\ ${ }^{a}$ Institute of Theoretical Science, University of Oregon \\ Eugene, Oregon 97403 \\ ${ }^{b}$ Physics Department, University of California, Davis \\ Davis, California 95616
}

\begin{abstract}
The 'WIMP miracle' for the relic abundance of thermal dark matter motivates weak scale dark matter with renormalizable couplings to standard model particles. We study minimal models with such couplings that explain dark matter as a thermal relic. The models contain a singlet dark matter particle with cubic renormalizable couplings between standard model particles and 'partner' particles with the same gauge quantum numbers as the standard model particle. The dark matter has spin $0, \frac{1}{2}$, or 1 , and may or may not be its own antiparticle. Each model has 3 parameters: the masses of the dark matter and standard model partners, and the cubic coupling. Requiring the correct relic abundance gives a 2-dimensional parameter space where collider and direct detection constraints can be directly compared. We focus on the case of dark matter interactions with colored particles. We find that collider and direct detection searches are remarkably complementary for these models. Direct detection limits for the cases where the dark matter is not its own antiparticle require dark matter masses to be in the multi-TeV range, where they are extremely difficult to probe in collider experiments. The models where dark matter is its own antiparticle are strongly constrained by collider searches for monojet and jets + MET signals. These models are constrained by direct detection mainly near the limit where the dark matter and partner masses are nearly degenerate, where collider searches become more difficult.
\end{abstract}




\section{Introduction}

The existence of dark matter is the strongest evidence we have for physics beyond the standard model, and it is a striking fact that a neutral particle with a mass at the weak scale with renormalizable couplings to standard model particles has a thermal relic density of order the observed value. This 'WIMP miracle' is a strong hint that motivates searches for direct detection of dark matter as well as dark matter production at colliders. In the coming years, dark matter direct and indirect detection experiments will reach new frontiers of sensitivity, and the LHC will begin operation at $14 \mathrm{TeV}$ after a very successful $8 \mathrm{TeV}$ run. These experiments will provide a crucial test of these ideas, and there is good reason to expect spectacular discoveries.

Naturalness also gives a motivation for new physics beyond the standard model that has been very influential in particle physics. The best-motivated and most successful framework for physics beyond the standard model is supersymmetry (SUSY). Among the attractive features of SUSY is that it contains a natural WIMP candidate, the lightest supersymmetric particle (LSP). Because of this, most of the work on the connection between direct detection and collider searches for dark matter have focused on SUSY. However, there is currently no signal for SUSY at the LHC, and minimal versions of SUSY must be fine-tuned to accommodate the observed Higgs mass and the null results of SUSY searches. Also, LSP dark matter is only viable for special regions of parameter space: either near maximal mixing, co-annihilation, or resonant annihilation. All of these mechanisms require special relations between unrelated parameters. More generally, the absence of any signal for physics beyond the standard model at the LHC has led many to question whether naturalness is in fact realized in nature, with or without SUSY.

These considerations motivate a more phenomenological approach to dark matter, one which assumes only the minimal extension of the standard model required to account for dark matter. One such approach that received wide attention is that of 'effective dark matter' 11 10]. In this approach, one assumes that the only new degrees of freedom relevant for dark matter phenomenology are the dark matter particles themselves. The only allowed interactions between the dark matter particles and the standard model particles are non-renormalizable interactions of the form

$$
\mathcal{L} \sim \frac{1}{M^{n}}|\mathrm{SM}|^{2}|\mathrm{DM}|^{2}
$$

where SM and DM denote standard model and dark matter fields, respectively, and $M$ is a mass scale that parameterizes the strength of the interaction. The important point that these same operators parameterize direct detection and monojet signals 
at colliders was made in Refs. [5 7]. For other work related to this approach, see e.g. Refs. [11-15]. This approach has many attractive features, but also several drawbacks. First, the 'WIMP miracle' that motivates weak scale dark matter is not built in. Also, collider bounds on higher dimension operators typically probe scales $M$ of order the energy of the collisions, so the UV completion of the operator becomes relevant for the collider phenomenology.

In this paper, we propose a different phenomenological approach to WIMP dark matter that addresses these issues. Motivated by the 'WIMP miracle,' we assume that dark matter has renormalizable interactions with standard model fields. We assume that the dark matter is a standard model gauge singlet. The alternative is that the dark matter is the neutral component of an electroweak multiplet, but this is highly constrained by direct detection experiments. Such models are viable models of dark matter only if the multiplet has $Y=0$ and the dark matter mass is in the $\mathrm{TeV}$ range [16]. For singlet dark matter, the only renormalizable couplings to standard model particles are quartic couplings of the Higgs boson to scalar dark matter $[17-20]$. This model has been extensively studied, and we only comment on it briefly below. Any other model with WIMP dark matter must contain additional degrees of freedom. We therefore consider cubic couplings of the form

$$
\Delta \mathcal{L} \sim \lambda(\mathrm{SM})(\widetilde{\mathrm{SM}})(\mathrm{DM}),
$$

where $\lambda$ is a dimensionless coupling. Here $\widetilde{\mathrm{SM}}$ is an additional field with the same gauge quantum numbers as the standard model field, so we call it a 'partner' field. This interaction is invariant under a $Z_{2}$ symmetry under which the dark matter and partner fields are odd, and therefore preserves the stability of the dark matter particle as long as the dark matter particle is lighter than the partner particle.

We focus on the case of interaction with colored standard model particles, since this is the case of most relevance to both the LHC and direct detection experiments. This means that the partner fields are colored, and can therefore be studied at the LHC. We do not consider the case where the colored standard model particle is the gluon because renormalizable interactions of this kind require embedding $S U(3)_{C}$ into a larger gauge group at the weak scale, and therefore require significantly more structure. We also assume that the interaction is invariant under the electroweak gauge group 1 This type of interaction is familiar from SUSY, where the partners are the superpartners, but we see that it has a direct phenomenological motivation.

\footnotetext{
${ }^{1}$ Interactions with dimensionless couplings that violate electroweak gauge symmetry can arise by integrating out $\mathrm{TeV}$ scale particles whose masses break electroweak symmetry due to couplings to the Higgs field. This requires additional structure, so we do not consider it on the grounds of minimality.
} 
Interactions of the form Eq. (1.2) have potentially serious problems with flavor physics, a fact that is also familiar from SUSY. For a generic flavor structure, loops involving virtual dark matter and partner fields with weak-scale masses will give rise to flavor-changing processes in conflict with experiment. The simplest solution to these constraints is to assume that the couplings and masses of the partner fields are approximately flavor-independent. The flavor constraints are most stringent for couplings to the first two generations of quarks, so we will consider three cases: $(i)$ 3 generations of quark partners with approximately equal couplings and masses; $(i i)$ 2 generations of quark partners coupling to the first 2 generations of quarks with approximately equal couplings and masses; ( $i i i)$ a single quark partner coupling to third generation quarks. To avoid proliferation of similar cases, we consider only the case of coupling to left-handed quark doublets. ${ }^{2}$ From now on, we denote the dark matter particle by $\chi$, the left-handed quark fields by $q$, and the quark partners by $Q$. We consider dark matter with spin $0, \frac{1}{2}$, or 1 , and the case where the dark matter is and is not its own antiparticle. Strictly speaking, the case of vector dark matter falls outside our minimal classification, since renormalizable interactions of gauge fields will not connect quark fields and partner fields. Generating such an interaction requires an extension of the electroweak gauge group as well as additional Higgs fields. We however include this case because it is phenomenologically similar to the others, and the real vector case arises in universal extra dimension models [21,22]. The quark partner fields are required to have masses that are invariant under electroweak symmetry. This means that the partners are complex scalars for fermion dark matter, and Dirac fermions for bosonic dark matter. We use 2-component spinor notation, so the Dirac mass for fermionic quark partners is written $Q^{c} Q$. The models are listed in Table 1 .

Because of the degeneracy assumed to avoid flavor constraints, each of these models has 3 parameters: a dark matter mass $m_{\chi}$, a partner mass $m_{Q}$, and a dimensionless coupling strength for the new cubic interaction $\lambda$. Since the motivation for these models is the 'WIMP miracle', we impose the constraint that the thermal dark matter density is the observed value. This results in a 2-dimensional parameter space that can be parameterized by the masses $m_{Q}$ and $m_{\chi}$. We have $m_{Q}>m_{\chi}$ by the assumption that the $\chi$ particle is stable. As with the effective dark matter models, we can directly compare collider, direct detections, and indirect detection constraints in a

\footnotetext{
${ }^{2}$ One case that could be significantly different is if the dark matter couples only to the righthanded top quark. However, the collider constraints come from top squark searches, which are similar in strength to bottom squark searches. The direct detection constraints are also qualitatively similar to the constraints for dark matter coupling to third generation left-handed quarks. Thus, the limits on that case will be similar to our models coupling to the left-handed third generation quarks.
} 


\begin{tabular}{|l|l|l|}
\hline \multicolumn{2}{|c|}{ Model Particles } & \multirow{2}{*}{$\mathcal{L}_{\text {int }}$} \\
\hline Dark matter $\chi$ & Quark partner $Q$ & $\lambda(\chi q) Q^{*}+$ h.c. \\
\hline \hline Majorana fermion & Complex scalar & $\lambda(\chi q) Q^{*}+$ h.c. \\
\hline Dirac fermion & Complex scalar & $\lambda\left(Q^{c} q\right) \chi+$ h.c. \\
\hline Real scalar & Dirac fermion & $\lambda\left(Q^{c} q\right) \chi+$ h.c. \\
\hline Complex scalar & Dirac fermion & $\lambda\left(q^{\dagger} \bar{\sigma}^{\mu} Q\right) \chi_{\mu}+$ h.c. \\
\hline Real vector & Dirac fermion & $\lambda\left(q^{\dagger} \bar{\sigma}^{\mu} Q\right) \chi_{\mu}+$ h.c. \\
\hline Complex vector & Dirac fermion \\
\hline
\end{tabular}

Table 1. Overview of the models considered in this paper. Spinors are written in 2-component notation. Here $q$ is the left-handed quark doublet of the standard model, $Q$ is the quark partner field, and $\chi$ is the dark matter field.

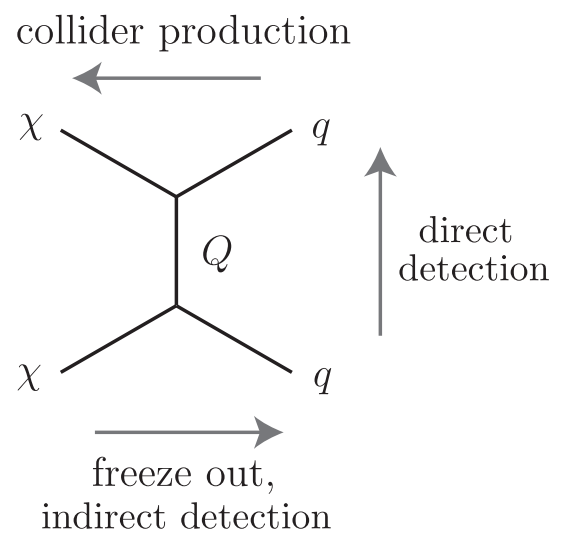

Fig. 1. Feynman diagram contributing to dark matter freeze-out, direct and indirect dark matter detection, and collider production of dark matter.

2-dimensional parameter space.

The annihilation of dark matter in the early universe, indirect detection of dark matter, and direct detection of dark matter are all dominated by the exchange of a partner particle, as shown in Fig. 1. The same diagram also gives rise to dark matter production at colliders, with an additional radiative particle required to tag the final state. This strongly motivates monojet searches at the LHC as a way to search for dark matter. In the present models, there are additional contributions to monojet final states, as shown in Fig. 2. In addition, there are jets plus missing energy signals from diagrams such as Fig. 3. These models therefore have a very rich phenomenology controlled by a simple 2-dimensional parameter space.

These models can be used in a number of different ways. First, we advocate 

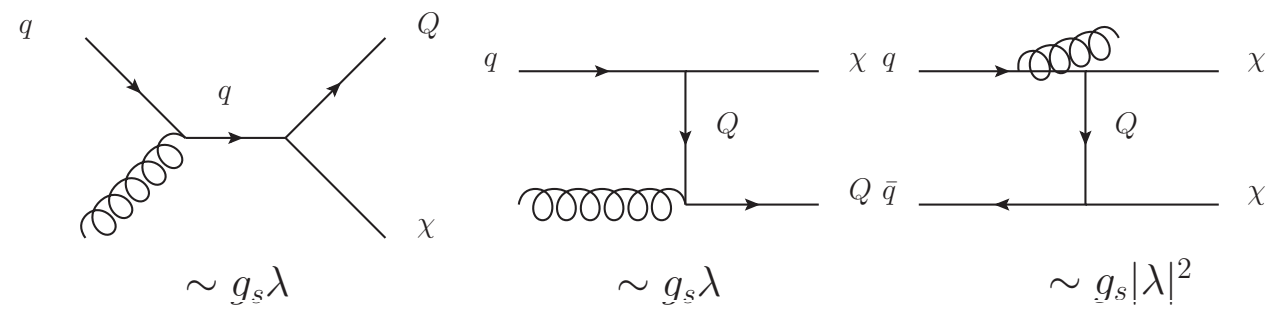

Fig. 2. Feynman diagrams contributing to monojet signals at a hadron collider.
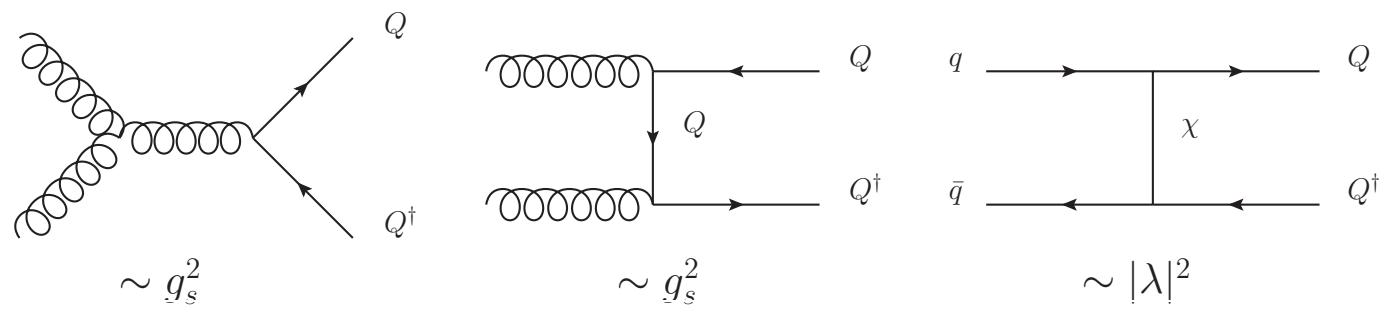

Fig. 3. Feynman Diagrams contributing to jets plus missing energy signals at a hadron collider. For scalar quark partners $Q$, there is an additional diagram involving the gluon- $Q$ quartic interaction that is not shown.

that they should be taken seriously as phenomenologically-motivated models of dark matter under the assumption that a small number of states is relevant. Another point of view comes from the fact that these models are also the minimal ones that can explain an excess in collider searches for jets plus missing energy, perhaps the most promising channel for the discovery of SUSY. If a signal is seen in jets+MET, it would immediately raise the question of whether WIMP dark matter is being produced in these events. In the context of the models we are considering, the rate and kinematics of such a signal would point to a specific region of the parameter space, which can be additionally probed by both monojet searches and direct detection experiments. A confirmation of the model predictions is clearly interesting, while ruling out the model tells us that additional states are required if the missing energy is due to WIMP dark matter. Finally, these models can be viewed as 'simplified models' 23 that parameterize the constraints of experiments in terms of a model with only the ingredients relevant for the signal. In this case, they provide a well-defined mapping between collider and astrophysical constraints on dark matter based on a well-defined set of physical assumptions. From all of these points of view, we believe these models can provide insight into the complementarity between these different approaches to testing the WIMP hypothesis.

Our main conclusion is that collider and direct detection experiments are remark- 
ably complementary under the assumptions we are making. Models where the dark matter is not its own antiparticle have unsuppressed direct detection cross sections, and current direct detection limits require the dark matter mass to be in the multi$\mathrm{TeV}$ range, where they are extremely difficult to probe with colliders. Models where the dark matter is its own antiparticle have suppressed direct detection cross section, and direct detection limits are strong near the degenerate limit $m_{\chi} \simeq m_{Q}$. In this regime, direct detection is enhanced by small energy denominators, while collider searches become more difficult due to smaller missing energy. Away from the degenerate limit, LHC searches are very constraining. Requiring the correct relic abundance gives a large (but still perturbative) value of the new cubic coupling, which means that production by $t$-channel $\chi$ exchange is dominant in a large region of parameter space. For monojets there is a substantial contribution due to associated production of $Q \chi$. Using existing monojet and jets + MET searches, we find that jets plus missing energy searches generally provide the strongest collider constraint. Due to the new processes, there could be improvements. Because the kinematics of $t$-channel exchange differs from that of standard colored production, we advocate the use of simplified models that include the $t$-channel contributions in LHC searches. Similarly, modifications to monojet searches could have enhanced sensitivity since the monojet's $p_{T}$ has a broad peak due to the two body decay of $Q$.

We end this introduction by commenting briefly on other phenomenological models with renormalizable interactions with dark matter that have been considered in the literature. One is a 'Higgs portal' [24] interaction of the form $S^{2} H^{\dagger} H$ between a singlet dark matter scalar $S$ and the Higgs field $H$ [17 20]. There are two regions of parameter space where this model gives a viable dark matter phenomenology. The first is the resonant annihilation region $m_{S} \simeq \frac{1}{2} m_{h}$, which can be probed both at collider and direct detection experiments, and a region with $m_{S} \gtrsim 80 \mathrm{GeV}$ that can be probed by direct detection, but is difficult to probe at colliders because $h \rightarrow S S$ is forbidden [25]. Another renormalizable phenomenological model is obtained by assuming that the dark matter is the electrically neutral component of an electroweak multiplet [16]. In order to avoid being ruled out by direct detection by $Z$ exchange these multiplets must have vanishing hypercharge. To get the right thermal relic abundance the dark matter must be at the $\mathrm{TeV}$ scale, and these models contain no colored particles, so these models are very difficult to probe at colliders. By contrast, the models considered here where the dark matter is its own antiparticle are viable for a wide range of parameters that will be extensively probed in both collider searches and direct dark matter searches.

An earlier work with a similar approach to ours considered only Majorana dark 
matter [26]. Our analysis goes beyond Ref. [26] by considering all allowed spins of the dark matter and quark partners and by considering additional collider processes.

This paper is organized as follows. In $\$ 2$, we discuss the constraints the models from the relic density, and direct and indirect detection and collider bounds. In $\S 3$ we present our main results, and our conclusions are presented in $\$ 4$. The appendices contain detailed formulas used to obtain our results.

\section{General Features}

In this section we consider the general features of the constraints on the models from dark matter relic abundance, direct and indirect detection, and LHC searches. We also discuss the effective operators that describe the interactions in the limit $m_{Q} \gg m_{\chi}$, since these allow one to understand many of the qualitative features of the models. We also consider the suppression and enhancement effects when the dark matter is nearly degenerate with the quark partner.

\subsection{Relic Abundance}

The relic abundance of non-baryonic matter is very accurately determined by cosmological constraints to be $\Omega_{\chi} h^{2}=0.1199 \pm 0.0027$ [27]. We assume that the dark matter is entirely composed of the $\chi$ particle in our model. Under the assumption that $\chi$ particles were in thermal equilibrium in the early universe, its present relic density is determined by freeze-out from the annihilation process $\chi \bar{\chi} \rightarrow q \bar{q}$ shown in Fig. 1. The relic abundance is determined by the thermally averaged annihilation cross section $\langle\sigma(\chi \bar{\chi} \rightarrow \bar{q} q) v\rangle$ at temperatures $T_{f} \sim m_{\chi} / 25$. The dark matter velocity is then $v^{2} \sim 0.1$, so we can expand

$$
\sigma(\chi \bar{\chi} \rightarrow \bar{q} q) v=a+b v^{2}+O\left(v^{4}\right)
$$

Approximate formulas for the relic density in terms of these parameters are given in Appendix A. The coefficients $a$ and $b$ represent $s$-wave and $p$-wave contributions, and can be computed in each model. Formulas for these are given in Appendix B.

As explained in $\$ 2.3$ below, the $s$-wave coefficient $a$ is suppressed by $m_{q}^{2} / m_{\chi}^{2}$ in the cases where the dark matter is a scalar or Majorana fermion. This arises because the dark matter couples only to left-handed quarks in our model. The $p$-wave coefficient $b$ is suppressed by $m_{q}^{2} / m_{\chi}^{2}$ only in the case of real scalar dark matter. Often the quark mass suppression of the $s$-wave is more severe than the velocity suppression of the $p$-wave which leads to larger couplings that vary rapidly with $m_{\chi}$. If these models 
couple to the top quark, the coupling $\lambda$ required to get the right relic abundance drops sharply for $m_{\chi}>m_{t}$ as the annihilation to top quarks becomes a viable channel.

These suppressions play a very important role in the phenomenology of these models, so it is worth considering the question of how robust this structure is. We have assumed that dark matter couples to left-handed quarks, but this is not particularly motivated over the assumption that the dominant coupling is to right-handed quarks. As long as we assume that only one quark multiplet dominates the phenomenology, there will be a similar chiral suppression, and the results will be qualitatively the same. We can avoid this suppression only if the quark partners for left- and right-handed quark fields have similar mass, including a large Dirac mass mixing them. Such a mass breaks electroweak symmetry, and therefore must arise from couplings to the Higgs. These couplings are constrained by precision electroweak measurements, and also require significant additional structure compared to the models we consider here. We conclude that the chiral suppression structure in these models is well-motivated by minimality. $!^{3}$

\subsection{Direct Detection of Dark Matter}

Direct detection experiments look for nuclear recoil events due to galactic dark matter. With standard assumptions about the dark matter halo distribution, the limits on signal events are interpreted as limits on the DM-nucleus elastic recoil cross section. For a review of direct detection theory see [29]. A useful method to determine the nuclear recoil cross section is to find the effective Lagrangian by integrating out the mediating particle, here $Q$ and computing the lowest order cross section. The connection between effective operators and the direct detection signal rate has been emphasized in recent work [2,3]. Matrix elements of the effective operators are typically either proportional to the spin of the nucleon or add coherently for each nucleon, and for this reason cross sections arising from these operators are called spin dependent (SD) or spin independent (SI), respectively. The coherent scattering for spin-independent cross sections leads to an $A^{2}$ enhancement of $\mathcal{O}\left(10^{4}\right)$, producing the most stringent limits. In our results, we present results for SI interactions only, as we find that they provide stronger limits than SD interactions. Constraints from SI couplings are also less sensitive than those from SD couplings to the flavors of the quarks that the dark matter interacts with. In all of our models, SI interactions are generated at some level, allowing us to focus on the current best SI limits from XENON100's 225

\footnotetext{
${ }^{3}$ In many models, these mixing masses are proportional to the quark masses in order to preserve minimal flavor violation [28, which again leads to a chiral suppression.
} 
$\mathrm{kg}$ · day run [30]. To see how this will improve in the future, we also will add projected sensitivities for LUX and XENON1T taken from DMtools [31].

Interestingly, current direct detection constraints are so strong that they put some of the models well beyond the reach of the LHC. For SI nucleon scattering, the quark operator with the largest matrix element is the vector current. By Lorentz invariance, the effective interaction coupling to the quark vector current can be a vector or pseudovector in the dark matter sector, with only the vector-vector coupling being unsuppressed in the nonrelativistic limit. Whether a dark matter vector operator is allowed depends on the dark matter quantum numbers. Complex dark matter models cannot forbid this vector coupling and thus have stringent constraints from XENON100 requiring multi-TeV masses. In models where the dark matter is its own antiparticle, the vector dark matter operators vanish or are a total derivative. Integrating by parts the total derivative changes the quark vector current into the mass operator which has a smaller matrix element. Thus in these models, much smaller masses for the dark matter are allowed.

\subsection{Heavy Partner Limit}

If the quark partners are much heavier than the dark matter particle, then both freezeout and direct detection can be described by contact interactions between dark matter and standard model particles obtained by integrating out the quark partners. Even for $m_{\chi} \sim m_{Q}$, annihilation is still described to a reasonable approximation by this contact interaction because the freeze-out temperature is $T \sim m_{\chi} / 25$. It can break down for direct detection however because the quark partner can go on shell in the limit $m_{Q} \rightarrow m_{\chi}$. Nonetheless, the structure of the effective interactions determines many important features of freeze-out and direct detection scattering rates, so we will describe them here. The main results are summarized in Table 2, Our discussion in this section will be qualitative. Precise formulas that are valid for general parameters are given in Appendix B.

Fermion dark matter: In this case, $Q$ is a scalar. This is similar to SUSY, where $Q$ is a squark. Integrating out the scalar $Q$ gives the effective interaction

$$
\mathcal{L}_{\mathrm{eff}} \sim \frac{\lambda^{2}}{m_{Q}^{2}}\left(\chi^{\dagger} \bar{\sigma}^{\mu} \chi\right)\left(q^{\dagger} \bar{\sigma}_{\mu} q\right),
$$

where we have used a Fierz identity.

If $\chi$ is a Majorana fermion, then $\chi^{\dagger} \bar{\sigma}^{\mu} \chi$ is pure axial vector, while the quark current is vector minus axial vector. For the annihilation that sets the relic abundance, the 
$s$-wave cross section is suppressed by $m_{q}^{2} / m_{\chi}^{2}$, so the annihilation is dominantly $p$ wave. This can be understood from $C$ and $P$ symmetries as reviewed in [32]. For any fermion-antifermion pair, $C=(-1)^{L+S}, P=(-1)^{L+1}$. For a Majorana pair, $C=+1$, so the $s$-wave channel requires zero net spin, $S=0$, which gives $P=-1$ and thus a pseudoscalar initial state $J^{P C}=0^{-+}$. In our models, the dark matter only couples to left-handed quarks (and right-handed anti-quarks), so in the limit of zero quark mass, the final spin is $S=1$ giving $C=(-1)^{L+1}, P=(-1)^{L+1}$ and thus $C$ and $P$ are not conserved for any value of the final state $L$. Thus, $s$-wave annihilation into the quarkantiquark pair requires a helicity flip proportional to $m_{q}$. The $p$-wave does not suffer from such a suppression since the initial values are $S=1, L=1, C=+1, P=+1$. Thus, parametrically the annihilation cross section goes as

$$
\text { Majorana DM: } \quad \sigma_{\mathrm{ann}} \sim \lambda^{4}\left(\frac{m_{q}^{2}}{m_{Q}^{4}}+v^{2} \frac{m_{\chi}^{2}}{m_{Q}^{4}}\right) .
$$

For direct detection, the non-relativistic regime determines the size of the scattering cross section. With the interaction Eq. (2.2), the coupling to the axial component of the quark current gives a spin-dependent operator, while the mixed vector-axial coupling is suppressed in the non-relativistic limit. Taking into account the next order correction in the momentum dependence of the $Q$ propagator leads to spinindependent operators, $m_{q} q q^{c}$ and a twist-two quark operator. These operators have smaller matrix elements than the quark vector current, and therefore generally give weaker constraints. An important exception discussed in $\$ 2.6$ is that there is an enhancement in the degenerate limit due to the resonance when $m_{\chi} \sim m_{Q}$. Away from this limit, the direct detection constraints are easily satisfied for masses that can be probed at the LHC. Summarizing, for large $m_{Q}$, and assuming that the $s$-wave cross section is subdominant in the annihilation, the spin-independent cross section goes as

$$
\text { Majorana DM: } \quad \sigma_{\mathrm{SI}} \sim \lambda^{4} \frac{m_{p}^{4} m_{\chi}^{2}}{m_{Q}^{8}} \sim \frac{m_{p}^{4}}{m_{Q}^{4}} \sigma_{\mathrm{ann}},
$$

where $m_{p}$ is the proton mass.

If $\chi$ is a Dirac fermion, then the requirement of $C=+1$ no longer holds in the discussion of the annihilation that produces the relic abundance. This allows a vector-vector coupling which gives $s$-wave annihilation

$$
\text { Dirac DM: } \quad \sigma_{\mathrm{ann}} \sim \lambda^{4} \frac{m_{\chi}^{2}}{m_{Q}^{4}},
$$


and an unsuppressed spin-dependent interaction for direct detection of order

$$
\text { Dirac DM: } \quad \sigma_{\mathrm{SI}} \sim \lambda^{4} \frac{m_{p}^{2}}{m_{Q}^{4}} \sim \frac{m_{p}^{2}}{m_{\chi}^{2}} \sigma_{\mathrm{ann}} .
$$

Obtaining the correct relic abundance requires $\sigma_{\text {ann }} \sim$ pb, which given XENON100 limits 30 requires heavy dark matter $m_{\chi} \gtrsim 5 \mathrm{TeV}$, well out of the reach of LHC, or very light dark matter, $m_{\chi} \lesssim 10 \mathrm{GeV}$.

Scalar Dark Matter: In this case $Q$ is a Dirac fermion. Integrating out $Q$, we obtain an effective interaction

$$
\mathcal{L}_{\text {eff }} \sim \frac{\lambda^{2}}{m_{Q}^{2}} \chi^{\dagger} q^{\dagger} i \bar{\sigma}^{\mu} \partial_{\mu}(\chi q)=\frac{\lambda^{2}}{m_{Q}^{2}} \chi^{\dagger} \partial_{\mu} \chi q^{\dagger} i \bar{\sigma}^{\mu} q+O\left(m_{q}\right) .
$$

If $\chi$ is a real scalar, we can integrate by parts to write the interaction as

$$
\text { Real scalar DM: } \quad \mathcal{L}_{\text {eff }} \sim \frac{\lambda^{2}}{m_{Q}^{2}} \chi^{2} \partial_{\mu}\left(q^{\dagger} \bar{\sigma}^{\mu} q\right)
$$

The divergence of the left-handed quark current is proportional to $m_{q}$, which gives a suppression for light quarks. This means that the annihilation is always chirally suppressed and thus $s$ and $p$-wave are both chirally suppressed. The coupling to the quark mass operator also means that direct detection is suppressed. This gives the parametric scaling

$$
\text { Real scalar DM: } \quad \sigma_{\mathrm{SI}} \sim \lambda^{4} \frac{m_{p}^{4}}{m_{\chi}^{2} m_{Q}^{4}} \sim \frac{m_{p}^{4}}{m_{q}^{2} m_{\chi}^{2}} \sigma_{\mathrm{ann}} .
$$

This leads to strong constraints unless the top quark is kinematically accessible in dark matter annihilation.

If $\chi$ is a complex scalar, one can integrate by parts to find

$$
\text { Complex scalar DM: } \quad \mathcal{L}_{\text {eff }} \sim \frac{\lambda^{2}}{2 m_{Q}^{2}}\left(\chi^{\dagger} \stackrel{\leftrightarrow}{\partial}_{\mu} \chi\right)\left(q^{\dagger} \bar{\sigma}^{\mu} q\right)+O\left(m_{q}\right) .
$$

For annihilation, the dark matter operator vanishes in the limit $v \rightarrow 0$, so the annihilation remains chirally suppressed at $s$-wave, but not in the $p$-wave. The direct detection is unsuppressed since the interaction in Eq. 2.10 has a nonzero vectorvector component. This leads to the scaling

$$
\text { Complex scalar DM: } \quad \sigma_{\mathrm{SI}} \sim \lambda^{4} \frac{m_{p}^{2}}{m_{Q}^{4}} \sim \frac{m_{p}^{2}}{m_{\chi}^{2}} \sigma_{\mathrm{ann}},
$$

which again requires dark matter mass in the multi-TeV range or very light $\mathrm{GeV}$ range. 
Vector Dark Matter: In this case $Q$ is again a Dirac fermion. Integrating out $Q$, we obtain an effective interaction

$$
\mathcal{L}_{\mathrm{eff}} \sim \frac{\lambda^{2}}{m_{Q}^{2}} q^{\dagger} \bar{\sigma}^{\mu} \chi_{\mu}^{\dagger} i \sigma^{\nu} \partial_{\nu}\left(\chi_{\rho} \bar{\sigma}^{\rho} q\right)
$$

We can use the identity $\bar{\sigma}^{\mu} \sigma^{\nu} \bar{\sigma}^{\rho}=g^{\mu \nu} \bar{\sigma}^{\rho}-g^{\mu \rho} \bar{\sigma}^{\nu}+g^{\nu \rho} \bar{\sigma}^{\mu}-i \epsilon^{\mu \nu \rho \kappa} \bar{\sigma}_{\kappa}$ [33] to simplify this. For a real vector $\chi_{\mu}^{\dagger}=\chi_{\mu}$, so we obtain

$$
\text { Real vector DM: } \quad \mathcal{L}_{\text {eff }} \sim \frac{\lambda^{2}}{m_{Q}^{2}}\left[i \chi^{\mu} \chi^{\nu} q^{\dagger} \bar{\sigma}_{\mu} \overleftrightarrow{\partial}_{\nu} q+\tilde{F}_{\mu \nu} \chi^{\mu} q^{\dagger} \bar{\sigma}^{\nu} q\right]+O\left(m_{q}\right)
$$

For the relic abundance, the first term contains a twist-two quark interaction which has an unsuppressed $s$-wave contribution. For direct detection, taking the nonrelativistic limit, this twist-two component has a small matrix element, but again is enhanced near degeneracy. The second term does not give a large vector-vector interaction since the $\nu=0$ term is proportional to $\tilde{F}_{\mu 0}=\epsilon_{\mu 0 \rho \sigma} \partial^{\rho} A^{\sigma}$, which is suppressed by the momentum transfer of the dark matter. In addition, that term gives a spin-dependent interaction when $\nu$ is a spatial index. Given the unsuppressed relic abundance and the suppressed direct detection, we find

$$
\text { Real vector DM: } \quad \sigma_{\mathrm{SI}} \sim \lambda^{4} \frac{m_{p}^{4}}{m_{\chi}^{2} m_{Q}^{4}} \sim \frac{m_{p}^{4}}{m_{\chi}^{4}} \sigma_{\mathrm{ann}} .
$$

For a complex vector, using the sigma matrix identity one finds an allowed vectorvector coupling, $A_{\mu}^{\dagger} \partial_{\nu} A_{\mu} q^{\dagger} \bar{\sigma}^{\nu} q$, which gives unsuppressed rates for both annihilation and direct detection. This gives

$$
\text { Complex vector DM: } \quad \sigma_{\mathrm{SI}} \sim \lambda^{4} \frac{m_{p}^{2}}{m_{Q}^{4}} \sim \frac{m_{p}^{2}}{m_{\chi}^{2}} \sigma_{\mathrm{ann}}
$$

and again pushes the dark matter mass to several TeV or below $10 \mathrm{GeV}$.

\subsection{Indirect Detection}

Indirect detection experiments looking for dark matter annihilation or decay products in cosmic rays are another potential constraint on these models. Our models do not have Sommerfeld enhancement, so the annihilation cross section today is smaller than the thermal annihilation cross section $\left\langle\sigma_{\text {ann }} v\right\rangle=3 \times 10^{-26} \mathrm{~cm}^{3} / \mathrm{s}$ required for relic abundance. For the most part, indirect detection constraints on dark matter annihilation channels give upper bounds for cross sections that are larger than the 


\begin{tabular}{|c|c|c|c|}
\hline \multicolumn{2}{|c|}{ Model } & \multirow{2}{*}{ Relic Abundance } & \multirow{2}{*}{ Direct Detection } \\
\hline$\chi$ & Q & & \\
\hline Majorana fermion & Complex scalar & $\begin{array}{c}a \sim m_{q}^{2} \\
\lambda \sim 0.5-2\end{array}$ & $\begin{array}{c}\text { Suppressed } \\
\sigma_{\mathrm{SI}} \stackrel{m_{Q} \gg m_{\chi}}{\sim} \frac{m_{p}^{4}}{m_{Q}^{4}} \sigma_{\mathrm{ann}}\end{array}$ \\
\hline Dirac fermion & Complex scalar & $\lambda \sim 0.2-1$ & $\begin{array}{c}\text { Unsuppressed } \\
\sigma_{Q} \gg m_{\chi} \frac{m_{p}^{2}}{m_{\chi}^{2}} \sigma_{\text {ann }}\end{array}$ \\
\hline Real scalar & Dirac fermion & $\begin{array}{c}a, b \sim m_{q}^{2} \\
\lambda \sim 0.5-5\end{array}$ & $\begin{array}{c}\text { Suppressed if } m_{\chi}>m_{t} \\
\sigma_{\mathrm{SI}} \stackrel{m_{Q} \gg m_{\chi}}{\sim} \frac{m_{p}^{4}}{m_{q}^{2} m_{\chi}^{2}} \sigma_{\text {ann }}\end{array}$ \\
\hline Complex scalar & Dirac fermion & $\begin{array}{c}a \sim m_{q}^{2} \\
\lambda \sim 0.5-2\end{array}$ & $\begin{array}{c}\text { Unsuppressed } \\
\sigma_{\mathrm{SI}} \stackrel{m_{Q} \gg m_{\chi}}{\sim} \frac{m_{p}^{2}}{m_{\chi}^{2}} \sigma_{\mathrm{ann}}\end{array}$ \\
\hline Real vector & Dirac fermion & $\lambda \sim 0.05-0.5$ & $\begin{array}{c}\text { Suppressed } \\
\sigma_{\mathrm{SI}} \stackrel{m_{Q} \gg m_{\chi}}{\sim} \frac{m_{p}^{4}}{m_{\chi}^{4}} \sigma_{\text {ann }}\end{array}$ \\
\hline Complex vector & Dirac fermion & $\lambda \sim 0.07-0.7$ & $\begin{array}{c}\text { Unsuppressed } \\
\sigma_{\mathrm{SI}} \stackrel{m_{Q} \gg m_{\chi}}{\sim} \frac{m_{p}^{2}}{m_{\chi}^{2}} \sigma_{\text {ann }}\end{array}$ \\
\hline
\end{tabular}

Table 2. Overview of results for relic abundance and direct detection for the various models. 
thermal value, so these do not constrain our models. One exception is gamma ray limits from Fermi-LAT observation of dwarf galaxies. By stacking several observed galaxies, a limit stronger than the thermal cross section can be achieved for certain annihilation channels. The one that applies to our models is the constraints on the cross section for annihilation to $b \bar{b}$ pairs, which has been analyzed in [34, 35]. The precise limit on the dark matter mass for a thermal cross section with $100 \%$ annihilation to $b \bar{b}$ pairs has large uncertainties due to the dark matter distribution of these galaxies, but gives lower limit of about $60 \mathrm{GeV}$.

As we will see below, this region is already highly constrained by direct detection and collider searches in our models. In addition, for models where the dark matter couples to all quarks, the presence of other annihilation channels significantly weakens this bound. If the annihilation cross section $s$-wave component is not chirally suppressed (as in the Dirac fermion and real vector dark matter models), then the annihilation currently is evenly spread amongst all of the open channels, so that the $b \bar{b}$ rate is $\frac{1}{5}-\frac{1}{6} \times$ thermal. Whereas if the $s$-wave component is chirally suppressed and the $p$-wave is not (as in the Majorana fermion and complex scalar models), the $s$-wave is dominantly into the heaviest quarks available, but this is typically smaller than thermal since the $p$-wave cross section at freezeout was important in getting a large enough cross section for the relic abundance. Finally, for the real scalar model, both $s$ and $p$-waves are chirally suppressed. In this case, if the dark matter mass is between the bottom and top quark masses, it will have a nearly thermal cross section to annihilate into bottom quarks. Then we would have a dark matter lower limit from the stacked analysis, requiring $m_{\chi} \gtrsim 60 \mathrm{GeV}$. Finally, if the dark matter only couples to third generation quarks, we also expect to find a similar limit of $m_{\chi} \gtrsim 60$ $\mathrm{GeV}$.

\subsection{Limits from Collider Experiments}

Since the new particles are odd under a $Z_{2}$ parity, they are produced in pairs in colliders. Thus the primary production channels at the LHC are $p p \rightarrow Q Q^{\dagger}, Q Q$, $Q \chi, \chi \chi^{\dagger}$. Since $Q$ decays to $q \chi$, these channels produce signatures with missing transverse energy (MET) and 2,1, and 0 parton-level jets, respectively. The zero jet event would be invisible to the detector, but an additional initial state radiation jet can make $p p \rightarrow \chi \chi+$ jet a visible signal.

This leads to two primary detection signals: dijet + MET and monojet + MET. For the dijet + MET signal, we utilize two CMS simplified model searches for light generation squarks based on $11.7 \mathrm{fb}^{-1}\left[36\right.$ and $19.5 \mathrm{fb}^{-1}$ [37] luminosity at $8 \mathrm{TeV}$ which have cross section limits as a function of squark, neutralino mass. These are 
combined by taking the best limit of the two searches and will be described from now on as the CMS dijets+MET search. We will also use the bottom squark limits from the earlier analysis [36]. We also found that the latest CMS search for top squarks 38] sets similar limits to the bottom squark search, so we chose to omit it from our plots. For monojet sensitivities, we used the latest CMS search [10], which placed limits on the monojet cross section in different MET bins. For the monojet and light generation squark search, we only consider light quark and gluon final states as jets.

To determine the cross sections for our models, the event rates for the collider production of the quark partners was calculated at parton level at leading order using MadGraph5 v1.4.8.4 39] The MadGraph model files were generated using FeynRules v1.6.0 [40]. Since our trilinear interaction strength can be substantial, this allows us to take into account the important production mechanism of same sign quark partners via a $t$-channel exchange of the dark matter particle as well as the monojets due to associated production of $Q \chi$. To apply the CMS limit for squarks to our total cross section, we assume that the signal efficiencies for QCD production and the new $t$ channel process are similar. Close to the degeneracy line, initial state radiation plays a crucial role for the signal selection. Initial state radiation differs between gluon and quark initial states and thus the naive approach of applying the simplified model cross section limit could break down in this part of the parameter space.

The diagrams that contribute to the monojet signal are shown in Fig. 2, The $Q$ particle is pair produced at colliders primarily through gluon-gluon and quarkantiquark annihilation (see Fig. 3). Production through strong production is generally larger since the gluon dominates in the proton PDF. The exception is at large quark partner masses, where the up and down quark PDFs become larger than the gluon. For the models where same sign quark partners can be produced by a $t$-channel exchange of the dark matter (similar to the third diagram of Fig. 3 with $q q \rightarrow Q Q$ production instead), this enhances the production rate at large quark partner masses. Thus, the new channel allow our limits to extend beyond the CMS analysis, even though we are just using a leading order calculation.

\subsection{Near Degenerate Effects}

Near the degeneracy of $m_{Q}$ and $m_{\chi}$ direct detection rates can gain additional sensitivity. The enhancement of the direct detection cross section can be seen by considering

\footnotetext{
${ }^{4}$ The production cross sections obtained from MadGraph can vary up to about $25-50 \%$ depending on the factorization/renormalization scale used. We do not know the best scale given the new contributions due to the $\lambda$ interaction, so we have used the default scale in MadGraph.
} 
tree-level interactions with the nucleus. As shown in Fig. 1, nucleon scattering in the $s$-channel process leads to resonant enhancement when $\sqrt{s} \simeq m_{\chi}$ is close to $m_{Q}$. As emphasized in [41], this enhances the coefficients of the spin-independent operators, increasing their direct detection rates. Conversely, the collider searches lose sensitivity near degeneracy. In this regime, the small mass splitting leads to soft jet production and signal events are lost due to $p_{T}$ cuts. Hence, we expect direct detection to be complementary to collider bounds in this region.

Another signal that is enhanced in this region is the indirect detection signal of photons produced in dark matter collisions with protons in AGN jets [42, 43]. However, [43] showed that even when saturating the XENON100 bound and taking favorable AGN and dark matter parameters, that this signal was still out of current sensitivity of gamma ray telescopes.

Finally, for the relic abundance calculation, when $m_{Q} \simeq m_{\chi}$, both particles freeze out at approximately the same temperature, leading to coannihilation effects not considered in our analysis. Since the squarks tend to have stronger annihilation cross sections, this reduces the required $\lambda$ near degeneracy [44], weakening the direct detection limits. Rather than perform a detailed analysis of this special region, we will simply highlight this region where our approximations begin to break down and omit the region $m_{Q}<1.1 m_{\chi}$ from our results.

\section{Results}

We now present our results. The main conclusions of \$2 are summarized in Table 2. As described earlier, due to the constraint $\sigma_{\mathrm{SI}} \lesssim$ few $\times 10^{-45} \mathrm{~cm}^{2}$ from XENON100 [30] the models where spin-independent interactions are not suppressed have $\sigma_{\mathrm{SI}} \sim$ $\left(m_{p} / m_{\chi}\right)^{2} 10^{-36} \mathrm{~cm}^{2}$, which requires dark matter masses above a TeV or below $10 \mathrm{GeV}$. The light mass region is constrained by the CMS monojet search, leaving the multi$\mathrm{TeV}$ range masses as the only unconstrained region. Thus, over the parameter space, the Dirac fermion, complex scalar, and complex vector DM models are primarily probed by direct detection experiments and are essentially irrelevant for the LHC. We therefore focus on the remaining models, which can be probed both by the LHC and direct detection experiments.

\subsection{Majorana dark matter}

We begin with the case where the dark matter is a Majorana fermion. The results for the models where the dark matter couples to all generations and just the light 
quarks are presented in Figs. 4 and 5. We see that these models have a large region of parameter space allowed by current constraints.

There are several important features to note in these results. First, the CMS dijet + MET search gives the most stringent constraint, although the monojet searches are also sensitive. Note that the sensitivity extends to partner masses well above 1 $\mathrm{TeV}$. Our results are cut off at $1.2 \mathrm{TeV}$ because the CMS dijet search does not present results for quark partner masses above this value, even though they clearly have sensitivity there. The reason for the sensitivity to very large masses is that for large $m_{Q}$ the coupling $\lambda$ is getting large in order to produce the correct relic abundance. The coupling is still perturbative in most of the interesting region, however. In the plots this is indicated by a black region where $\lambda>3$, corresponding to a perturbative expansion parameter $\lambda^{2} / 8 \pi^{2} \sim 0.1$. The bound from the dijet + MET is strengthened considerably by the presence of the $t$-channel contribution proportional to $\lambda^{2}$. This is because Majorana dark matter allows $t$-channel production from the $q q$ (as opposed to $q \bar{q}$ ) initial state, which has a large PDF at large $x$. The dijet bounds we present are obtained by applying the cross section limit for the simplified model considered in the CMS analysis. We expect the sensitivity differences between QCD production and the new $t$-channel process to be most pronounced when the quark partner and dark matter are nearly degenerate due to the differences in initial state radiation off of gluons and quarks. Thus, we advocate that CMS and ATLAS present results for a simplified model with $t$-channel production to get accurate limits on this scenario.

The monojet searches are less sensitive, but they have substantial overlap with the dijet + MET search. The monojet limits are slightly weaker than the dijet limits but also extend to large values of $m_{Q}$, where the limits asymptote to the 'effective' dark matter' approach. In our models, the monojet signal will have a broad peak in the missing transverse energy spectrum around $\frac{m_{Q}^{2}-m_{\chi}^{2}}{2 m_{Q}}$, compared with the effective dark matter models, which have a falling, featureless enhancement. It may be interesting to investigate whether searches optimized for the models considered here will have significantly enhanced sensitivity.

For the case where dark matter couples only to third-generation quarks, production is due to QCD only and thus is more suppressed. The bottom quark partner results are shown in Fig. 6. (We also show the results of the third generation search for the case where the dark matter couples to all 3 generations in Fig. (4) There is presently no 'mono- $b$ ' search, but this would presumably be quite sensitive in this model.

The collider limits become much weaker as we approach the degenerate limit $m_{\chi} \simeq m_{Q}$, since this reduces the missing energy. However, in the region direct detec- 
tion has enhanced sensitivity, because the energy denominator suppressing the direct detection cross section is $m_{Q}-m_{\chi}$. In fact, the current XENON100 limit already rules out the entire region near the degenerate limit. In this region, co-annihilation becomes important, and this was not included in our relic abundance calculations, so these limits are not fully reliable. However, as can be seen in the XENON1T and LUX projections, the improvements in future years in spin-independent direct detection limits will push the sensitivity into a region where the coannihilation effects are negligible. The collider limits on the degenerate region are also expected to improve, so in future years we may expect direct detection and collider searches to fully probe this region.

Note that the direct detection bounds are very weak for $m_{\chi} \ll m_{Q}$. This is due to the fact that the spin-dependent cross section goes as $m_{Q}^{-4}$, as shown in Table 2 . This feature is not present in the other models considered below, so in these cases direct detection is more sensitive for $m_{\chi} \ll m_{Q}$.

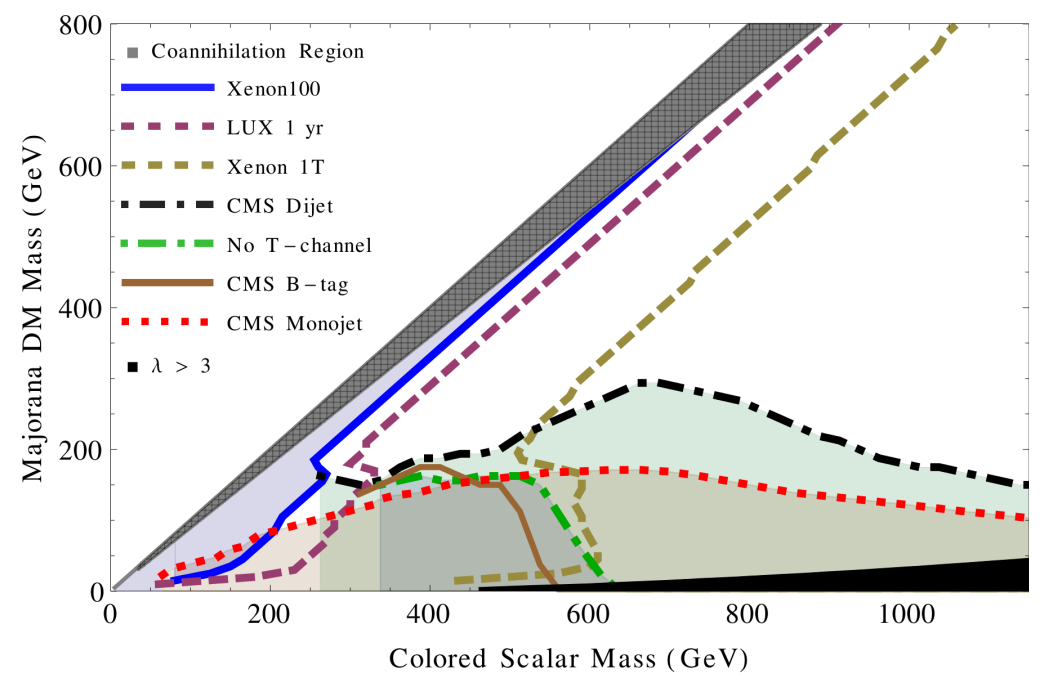

Fig. 4. Limits on Majorana dark matter coupling to all generations. The limits from the CMS dijet searches are shown with lines (black dot dashed, green dot dashed, brown solid) taking into account the production modes (all, QCD only, bottom quark) and the CMS monojet is shown in red dotted. The direct detection limits (XENON100 in blue solid, projected LUX and XENON1T in dashed) have an edge at $m_{\chi} \simeq m_{t}$ due to the effects of the top quark on the relic abundance. There are two regions where the results have large uncertainties. In the grey region $m_{Q}<$ $1.1 m_{\chi}$, coannihilation effects can strongly suppress $\lambda$, weakening the bounds. In the black region $m_{Q} \gg m_{\chi}, \lambda>3$ is required to obtain the correct relic abundance. 


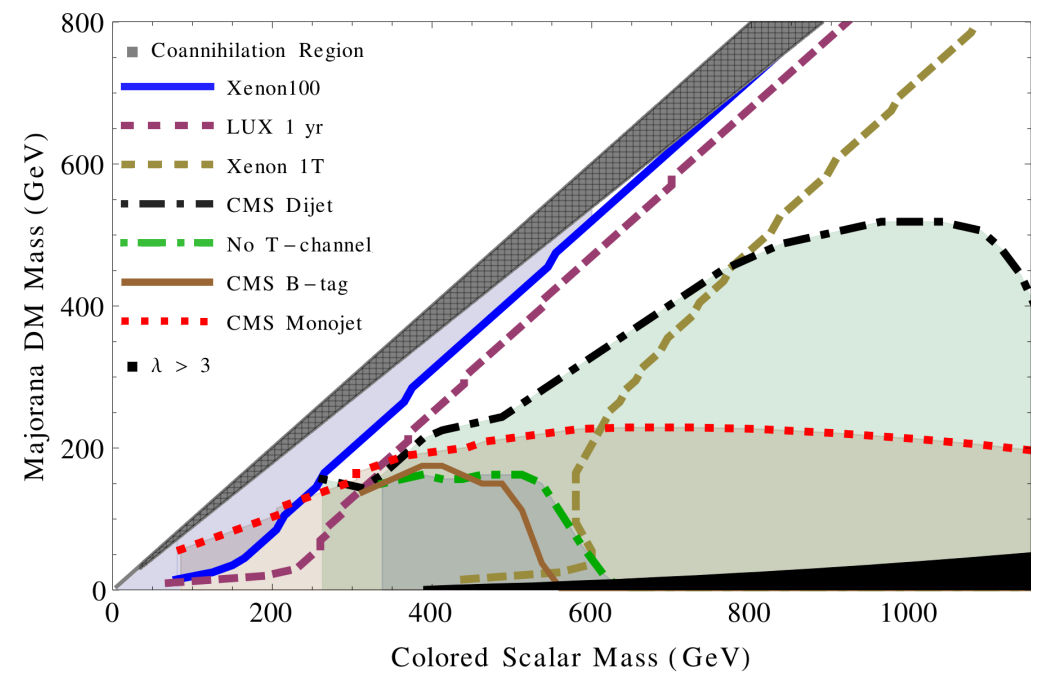

Fig. 5. Limits on Majorana dark matter coupling to the lightest two generations. Labeling as in Fig. 4.

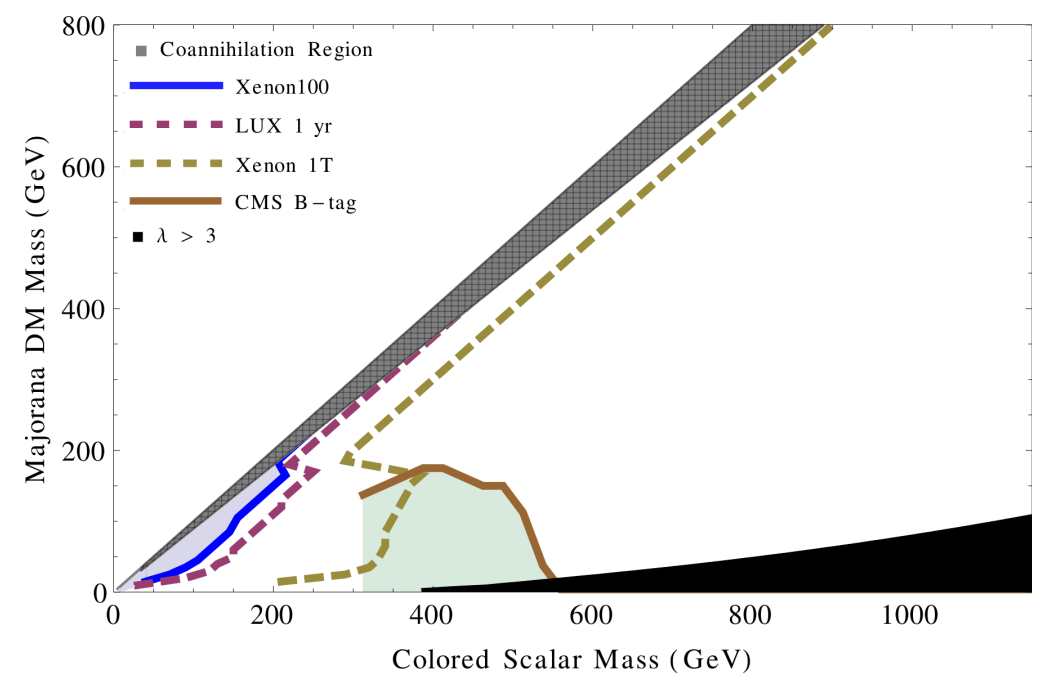

Fig. 6. Limits on Majorana dark matter coupling to third generation only. Labeling as in Fig. 4.

\subsection{Real scalar dark matter}

For this model, both the $s$ - and $p$-wave annihilation cross sections are chirally suppressed. Therefore, if the dark matter couples only to the lightest two generations, its interaction strength is required to be non-perturbatively strong to get the right relic abundance unless $m_{Q} \lesssim 400 \mathrm{GeV}$. However, this region is excluded by the XENON100 and CMS monojet limits. Thus, we present results only for the cases of 


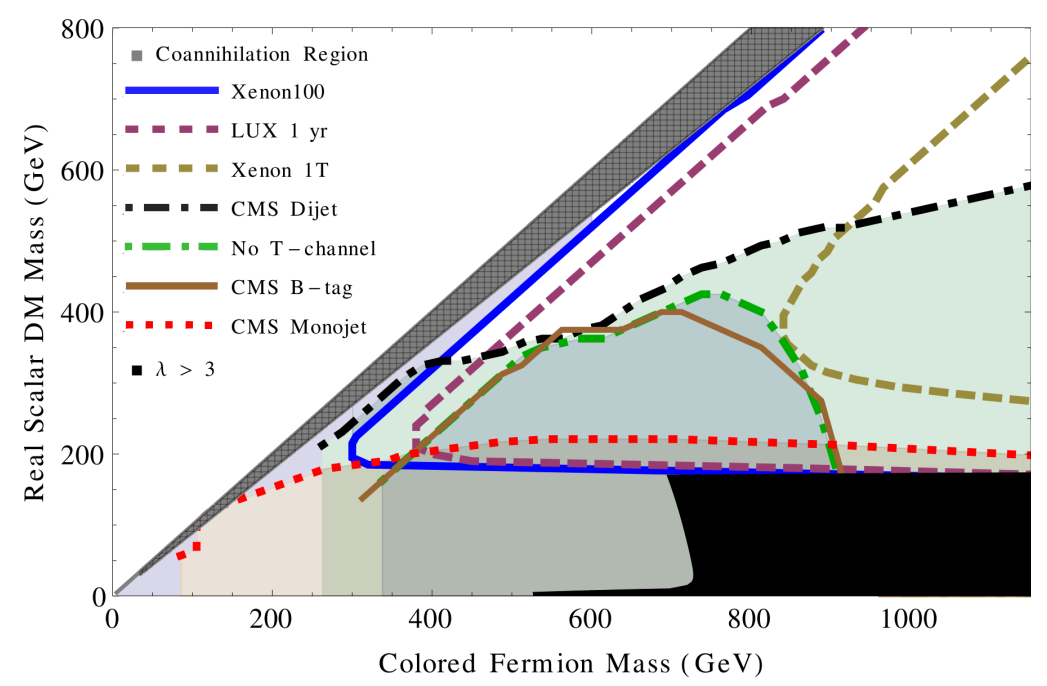

Fig. 7. Limits on real scalar dark matter coupling to all generations. Labeling as in Fig. 4

coupling to all generations and the third generation only. The results are shown in Figs. 7 and 8 . If $m_{\chi}<m_{t}$, the coupling $\lambda$ cannot account for the relic abundance unless $m_{Q} \lesssim 700 \mathrm{GeV}$.

Note that the CMS dijet limits are enhanced with respect to the Majorana models because fermion quark partners have a larger production cross section than scalar quark partners. The constraints using just the QCD production mechanism would already rule out quark partners up to about $1 \mathrm{TeV}$ for light dark matter. Including the $t$-channel, again extends the limit to higher masses.

In the models where dark matter couples to all generations, the XENON100 limit is comparable to the CMS monojet limit. This is a result of the relic abundance constraint: the value of $\lambda$ required to get the right relic abundance drops sharply once $m_{\chi}>m_{t}$.

\subsection{Real vector dark matter:}

For the real vector dark matter model, the interaction strength is small, since neither the $s$ and $p$-wave cross sections are chirally suppressed. The results are in Figs. 9, 10, and 11. These smaller couplings lead to weaker direct detection constraints than the real scalar dark matter case. Note the behavior of an asymptotic limit as $m_{Q} \gg m_{\chi}$ is explained by the fact that in this limit, $\sigma_{\mathrm{SI}}$ only depends on $m_{\chi}$ (see Table 2). On the other hand, the collider constraints are still strong due to the large cross section for fermion quark partners and the $t$-channel mechanism. The $t$-channel matrix element 


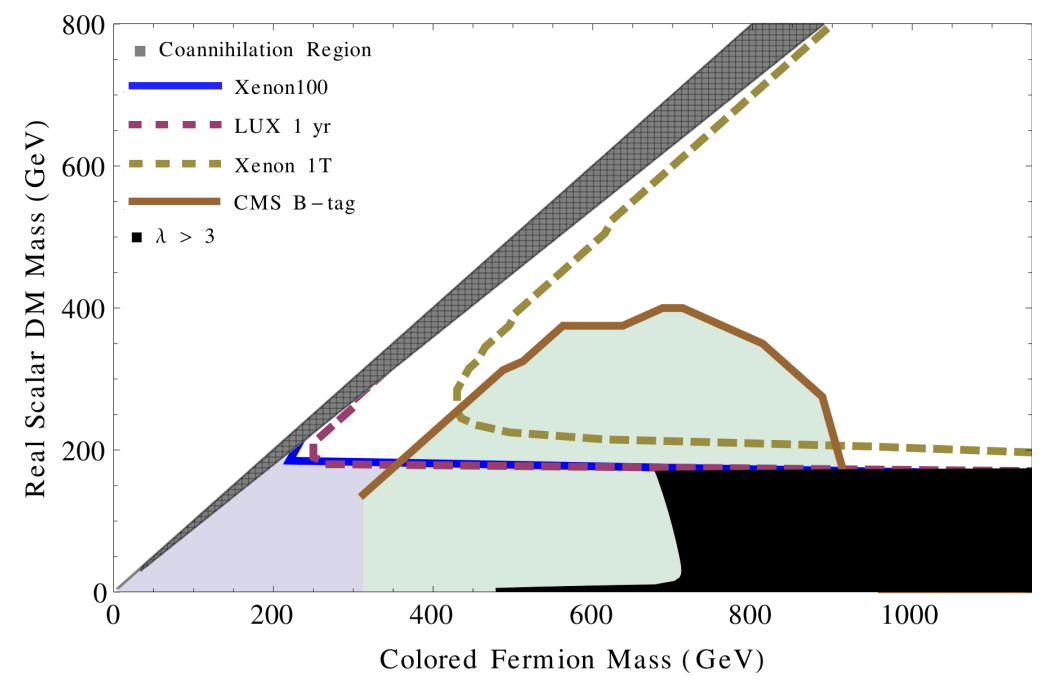

Fig. 8. Limits on real scalar dark matter coupling to third generation only. Labeling as in Fig. 4.

receives an enhancement of $\sim m_{Q}^{2} / m_{\chi}^{2}$ due to the $q^{\mu} q^{\nu} / m_{\chi}^{2}$ part of the dark matter propagator. This enhancement will be cut off by the Higgs sector responsible for giving a mass to the dark matter vector particle, and so the $t$-channel bound given here is too strong. In a complete model, the collider limit will be somewhere between the bounds with and without the $t$-channel contribution. The monojet bounds are not affected by this theoretical uncertainty, and these extend to large values of $m_{Q}$.

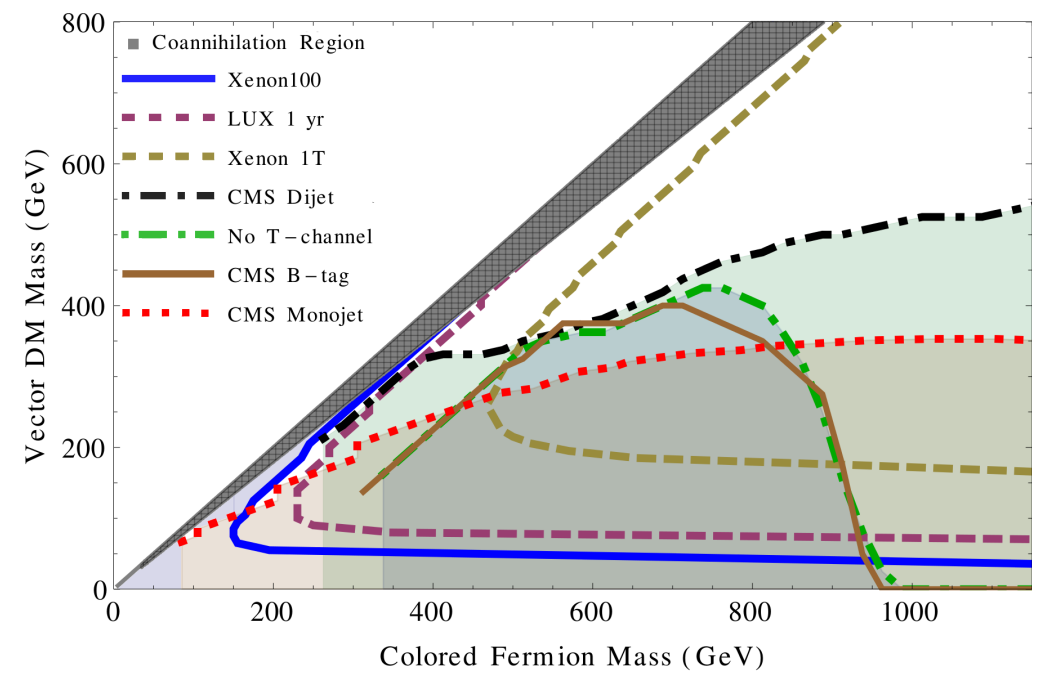

Fig. 9. Limits on real vector dark matter coupling to all generations. Labeling as in Fig. 4 . 


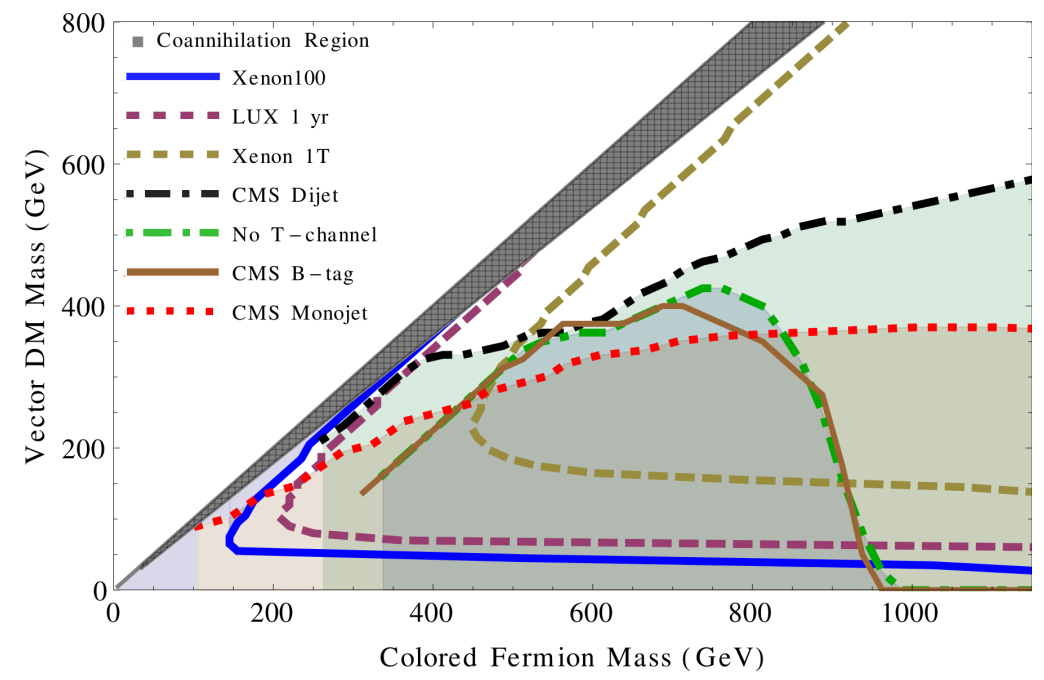

Fig. 10. Limits on real vector dark matter coupling to the lightest two generations. Labeling as in Fig. 4.

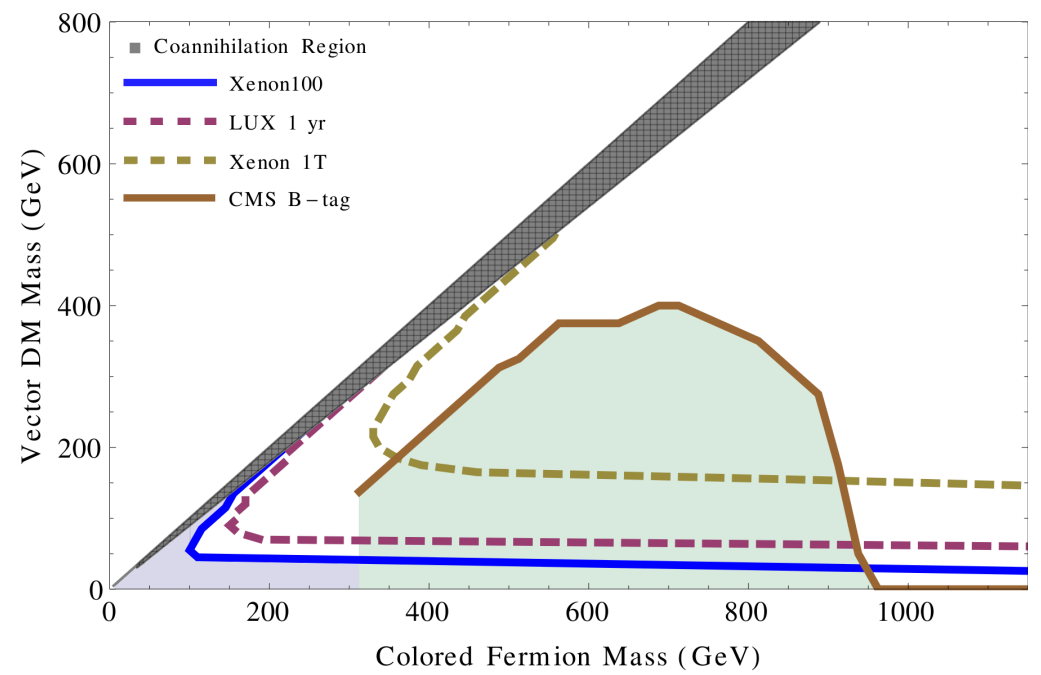

Fig. 11. Limits on real vector dark matter coupling to third generation only. Labeling as in Fig. 4 .

\section{Conclusions}

We have proposed and studied a new phenomenological approach to interpreting dark matter searches, based on a minimal particle content required to explain WIMP dark matter. The models consist of a singlet dark matter particle coupling to quarks and 'quark partners.' We consider dark matter with spin $0, \frac{1}{2}$, and 1 that is or is not its own antiparticle. In each case, imposing the constraint that the dark matter have 
the correct thermal relic abundance fixes the interaction strength, so the model is completely specified by the masses of the dark matter and quark partners.

These 'effective WIMP' models therefore have a 2-dimensional parameter space in which the reach of dark matter direct and indirect detection experiments can be directly compared to collider searches for missing energy events. This extends the approach of 'effective dark matter' models, and is complementary to more ambitious approaches based on complete models, such as scans for supersymmetric dark matter (e.g. [45]).

Our main results are as follows.

- The most sensitive direct detection constraints come from spin-independent interactions. Indirect detection is currently not competitive.

- The most sensitive collider constraints are from jets + MET searches and monojet searches, with the former generally more sensitive. The production cross sections at colliders are greatly enhanced by processes involving the new coupling, extending the reach for the colored states to very high masses. Collider searches for effective WIMPs may be improved by optimizing for these production modes.

- The direct detection and collider constraints are remarkably complementary. If the dark matter is not its own antiparticle, direct detection constraints require the dark matter mass to be in the multi-TeV range, far out of reach of LHC searches. If the dark matter is its own antiparticle, both collider and direct detection are sensitive. Direct detection has enhanced sensitivity in the degenerate region $m_{Q} \simeq m_{\chi}$ where collider searches are less sensitive due to reduced missing energy. On the other hand, collider searches generally probe a larger region of the parameter space away from the degenerate limit.

- Mono- $b$ searches can significantly enhance sensitivity to models where dark matter couples dominantly to third-generation quarks.

We advocate that these models can play an important role in interpreting searches for astrophysical dark matter and dark matter at colliders. They allow us to unambiguously compare both kinds of dark matter searches in the context of well-defined physical minimality assumptions. Of course these assumptions are strong ones, but they are crucial ones to test. If a signal is observed in either direct detection or collider searches, one of the most important questions to answer is whether the signal can be explained by a minimal number of additional states, or whether the dark matter 
is part of a larger sector of new particles (as in SUSY) that can be searched for at colliders. We have shown that effective WIMP models have multiple overlapping as well as complementary probes that can be unambiguously compared to help answer this question.

\section{Acknowledgements}

We thank Mariarosaria D'Alfonso, Eva Halkiadakis, David Stuart, and Frank Wuerthwein for discussions on the CMS Simplified Models. SC was supported in part by the Department of Energy under grant DE-FG02-13ER41986. RE, JH, and ML were supported in part by the Department of Energy under grant DE-FG02-91ER40674.

\section{Appendix A: Relic Abundance and Direct Detection Numerics}

The relic abundance is given approximately by

$$
\begin{aligned}
\Omega_{\chi} h^{2} & \simeq 1.07 \cdot 10^{9} \mathrm{GeV}^{-1} \frac{x_{f}}{M_{\text {Planck }} g_{* S}^{1 / 2}\left(a+3 b / x_{f}\right)} \\
e^{x_{f}} & =c(c+2) \sqrt{\frac{45}{8}} \frac{m_{\chi} M_{\text {Planck }}\left(a+6 b / x_{f}\right)}{\pi^{3} g_{* S}^{1 / 2} x_{f}^{1 / 2}}
\end{aligned}
$$

where $x_{f}=m_{\chi} / T_{f} \sim 25$ is the inverse freeze-out temperature and $g_{* S}$ is the relativistic degrees of freedom. In Appendix B, we list the formulas for $a, b$ for the models being considered. We use the values $c=\frac{1}{2}[46], g_{* S}=100, \Omega_{\chi} h^{2}=0.1199 \pm 0.0027$ [27]. To calculate the direct detection scattering cross section, we use the matrix element values in Table 3, where the numbers (and notation) are taken from [47]. 
Table 3. Parameters for quark and gluon matrix elements

\begin{tabular}{|l|c|}
\hline \multicolumn{2}{|c|}{ For proton } \\
\hline$f_{T u}$ & 0.023 \\
$f_{T d}$ & 0.032 \\
$f_{T s}$ & 0.020 \\
$f_{T G}$ & 0.925 \\
\hline \multicolumn{2}{|c|}{ For neutron } \\
\hline$f_{T u}$ & 0.017 \\
$f_{T d}$ & 0.041 \\
$f_{T s}$ & 0.020 \\
$f_{T G}$ & 0.922 \\
\hline
\end{tabular}

\begin{tabular}{|l|l||l|l|}
\hline \multicolumn{4}{|c|}{ Second moment at $\mu=m_{Z}$} \\
(for proton)
\end{tabular}

\section{Appendix B: Model Details}

In this appendix, we collect our calculations for the annihilation and direct detection cross sections for the different models. We describe the Majorana dark matter model in detail and summarize the results for the other models.

\section{B.1 Majorana Dark Matter}

In this model, the dark matter particle is a Majorana fermion and the quark partners are scalars. The Lagrangian for the new physics in two component notation is

$$
\mathcal{L}=\left|D_{\mu} Q\right|^{2}-m_{Q}^{2}|Q|^{2}+i \chi^{\dagger} \bar{\sigma} \cdot \partial \chi-\frac{1}{2} m_{\chi}\left(\chi^{2}+\chi^{\dagger 2}\right)+\lambda(\chi q) Q^{*}+\lambda^{*}\left(\chi^{\dagger} q^{\dagger}\right) Q
$$

\section{B.1.1 Relic Density}

The relic density is determined by the velocity-averaged annihilation cross section $\langle\sigma v\rangle$ which is commonly parametrized by the coefficients

$$
\langle\sigma v\rangle \simeq a+b v^{2}
$$

In this model, for the annihilation cross section $\chi \chi^{\dagger} \rightarrow q q^{\dagger}, a$ and $b$ are found to be

$$
a=\frac{3 m_{\chi}^{2} \sqrt{1-r} r \lambda^{4}}{32 \pi\left(m_{Q}^{2}-m_{\chi}^{2}(r-1)\right)^{2}},
$$




$$
\begin{aligned}
b= & \frac{\lambda^{4} m_{\chi}^{2}}{256 \pi\left(m_{Q}^{2}-m_{\chi}^{2}(r-1)\right)^{4} \sqrt{1-r}}\left[-2 m_{Q}^{2} m_{\chi}^{2} r\left(22-35 r+13 r^{2}\right)\right. \\
& \left.+m_{Q}^{4}\left(16-26 r+13 r^{2}\right)+m_{\chi}^{4}(r-1)^{2}\left(16-10 r+13 r^{2}\right)\right]
\end{aligned}
$$

where $r \equiv m_{q}^{2} / m_{\chi}^{2}$. Thus, in the massless quark limit, the $s$-wave vanishes and we have the leading order results

$$
\begin{gathered}
a \stackrel{r \rightarrow 0}{\simeq} \frac{3 m_{\chi}^{2} r \lambda^{4}}{32 \pi\left(m_{Q}^{2}+m_{\chi}^{2}\right)^{2}} \\
b \stackrel{r \rightarrow 0}{\simeq} \lambda^{4} \frac{m_{\chi}^{2}\left(m_{Q}^{4}+m_{\chi}^{4}\right)}{16 \pi\left(m_{Q}^{2}+m_{\chi}^{2}\right)^{4}}
\end{gathered}
$$

\section{B.1.2 Direct Detection}

After integrating out the colored partner (since the typical momentum transfer at direct detection experiments is small compared to its mass) we get the low-energy effective Lagrangian [41], which is written in four component notation as

$$
\begin{aligned}
\mathcal{L}_{\text {eff }}=\sum_{q}\left(f_{q} m_{q} \bar{\chi} \chi \bar{q} q\right. & d_{q} \bar{\chi} \gamma_{\mu} \gamma_{5} \chi \bar{q} \gamma^{\mu} \gamma_{5} q+\frac{g_{q}^{(1)}}{m_{\chi}} \bar{\chi} i \partial^{\mu} \gamma^{\nu} \chi \mathcal{O}_{\mu \nu}^{q}+\frac{g_{q}^{(2)}}{m_{\chi}^{2}} \bar{\chi}\left(i \partial^{\mu}\right)\left(i \partial^{\nu}\right) \chi \mathcal{O}_{\mu \nu}^{q} \\
& \left.+f_{G} \bar{\chi} \chi G_{\mu \nu}^{a} G^{a \mu \nu}+\frac{g_{G}^{(1)}}{m_{\chi}} \bar{\chi} i \partial^{\mu} \gamma^{\nu} \chi \mathcal{O}_{\mu \nu}^{g}+\frac{g_{G}^{(2)}}{m_{\chi}^{2}} \bar{\chi}\left(i \partial^{\mu}\right)\left(i \partial_{\nu}\right) \chi \mathcal{O}_{\mu \nu}^{g}\right)
\end{aligned}
$$

where $\mathcal{O}_{\mu \nu}^{q}$ and $\mathcal{O}_{\mu \nu}^{g}$ are the twist-2 operators for quarks and gluons:

$$
\begin{gathered}
\mathcal{O}_{\mu \nu}^{q} \equiv \frac{1}{2} \bar{q} i\left(D_{\mu} \gamma_{\nu}+D_{\nu} \gamma_{\mu}-\frac{1}{2} g_{\mu \nu} \not D\right) q \\
\mathcal{O}_{\mu \nu}^{g} \equiv\left(G_{\mu}^{a \rho} G_{\rho \nu}^{a}+\frac{1}{4} g_{\mu \nu} G_{\alpha \beta}^{a} G^{a \alpha \beta}\right)
\end{gathered}
$$

Then the spin-independent scattering cross section of DM with nucleons $(N=p, n)$ is obtained from the effective Lagrangian as

$$
\sigma_{\chi N}=\frac{4}{\pi} \mu_{N}^{2}\left|f_{N}\right|^{2}
$$

where $\mu_{N}$ is the reduced mass of the $\chi, N$ system. 
The SI effective coupling $f_{N}$ is evaluated using the nucleon matrix elements of quark and gluon operators giving:

$$
\frac{f_{N}}{m_{p}}=\sum_{q=u, d, s} f_{q} f_{T q}+\sum_{q=u, d, s, c, b} \frac{3}{4}(q(2)+\bar{q}(2))\left(g_{q}^{(1)}+g_{q}^{(2)}\right)-\frac{8 \pi}{9 \alpha_{s}} f_{T G} f_{G}
$$

where

$$
\begin{aligned}
& f_{q}=\frac{m_{\chi} \lambda^{2}}{16\left(m_{Q}^{2}-m_{\chi}^{2}\right)^{2}} \\
& g_{q}^{(1)}=\frac{m_{\chi}}{4} \frac{\lambda^{2}}{\left(m_{Q}^{2}-m_{\chi}^{2}\right)^{2}} \\
& g_{q}^{(2)}=0 \\
& f_{G} \simeq-\frac{\alpha_{s} m_{\chi} \lambda^{2}}{192 \pi m_{Q}^{2}\left(m_{Q}^{2}-m_{\chi}^{2}\right)}\left(\sum_{q=\text { all }} 1+\sum_{q=c, b, t} c_{q}\right)
\end{aligned}
$$

As explained in [47], the first sum in $f_{G}$ extends over all quarks coupling to the dark matter and is the short distance contribution, whereas the second sum is the long-distance contribution of heavy quarks and has a QCD correction factor $c_{q}=$ $1+11 \alpha_{s}\left(m_{q}\right) / 4 \pi$ [48]. Following [47], we take $\left(c_{c}, c_{b}, c_{t}\right)=(1.32,1.19,1)$. The factors of $1 /\left(m_{Q}^{2}-m_{\chi}^{2}\right)$ demonstrate the enhancement of the direct detection cross section when $Q, \chi$ become degenerate. In particular, the twist-two terms proportional to $g_{q}^{(1)}$ are strongly enhanced in this limit given the large values for $q(2)+\bar{q}(2)$.

\section{B.2 Dirac Dark Matter}

\section{B.2.1 Relic Density}

$$
\begin{gathered}
a=\frac{3 m_{\chi}^{2} \sqrt{1-r} \lambda^{4}}{32 \pi\left(m_{Q}^{2}-m_{\chi}^{2}(r-1)\right)^{2}} \\
b=\frac{\lambda^{4} m_{\chi}^{2}}{256 \pi\left(m_{Q}^{2}-m_{\chi}^{2}(r-1)\right)^{4} \sqrt{1-r}}\left[m_{Q}^{4}\left(8-7 r+2 r^{2}\right)\right. \\
\left.+m_{\chi}^{4}(r-1)^{2}\left(-8+9 r+2 r^{2}\right)+2 m_{Q}^{2} m_{\chi}^{2}\left(-12+13 r+r^{2}-2 r^{3}\right)\right]
\end{gathered}
$$

To lowest order in $r=m_{q}^{2} / m_{\chi}^{2}$,

$$
a \stackrel{r \rightarrow 0}{\simeq} \frac{3 \lambda^{4} m_{\chi}^{2}}{32 \pi\left(m_{Q}^{2}+m_{\chi}^{2}\right)^{2}}
$$




$$
b \stackrel{r \rightarrow 0}{\simeq}-\frac{\lambda^{4} m_{\chi}^{2}\left(-m_{Q}^{4}+3 m_{Q}^{2} m_{\chi}^{2}+m_{\chi}^{4}\right)}{32 \pi\left(m_{Q}^{2}+m_{\chi}^{2}\right)^{4}}
$$

so the cross section is not $s$-wave suppressed.

\section{B.2.2 Direct Detection}

We get a low energy effective Lagrangian

$$
\left.\mathcal{L}_{\text {eff }} \sim \frac{\lambda^{2}}{8\left(m_{\chi}^{2}-m_{Q}^{2}\right)}\left(\bar{q} \gamma_{\mu} q \bar{\chi} \gamma^{\mu} \chi-\bar{q} \gamma_{\mu} \gamma^{5} q \bar{\chi} \gamma^{\mu} \gamma^{5} \chi\right)\right)
$$

The vector-vector interaction gives a spin-independent cross section, which is only dependent on interactions to the up and down quarks. In our models, the coupling to up and down quarks is the same and following a few steps (see e.g. [49]) gives a cross section per nucleon

$$
\sigma_{\chi N}=\frac{9 \lambda^{4} \mu_{N}^{2}}{64 \pi\left(m_{\chi}^{2}-m_{Q}^{2}\right)^{2}}
$$

\section{B.3 Real Scalar Dark Matter}

\section{B.3.1 Relic Density}

$$
\begin{gathered}
a=\frac{3 m_{\chi}^{2}(1-r)^{3 / 2} r \lambda^{4}}{4 \pi\left(m_{Q}^{2}-m_{\chi}^{2}(r-1)\right)^{2}} \\
b=\frac{m_{\chi}^{2} \sqrt{1-r} r\left(9 m_{Q}^{4} r+m_{\chi}^{4}(r-1)^{2}(-16+9 r)-2 m_{Q}^{2} m_{\chi}^{2}\left(16-25 r+9 r^{2}\right)\right) \lambda^{4}}{32 \pi\left(m_{Q}^{2}-m_{\chi}^{2}(r-1)\right)^{4}}
\end{gathered}
$$

Both $a$ and $b$ vanish as $r \rightarrow 0$. To lowest order,

$$
\begin{gathered}
a \stackrel{r \rightarrow 0}{\simeq} r \frac{3 m_{\chi}^{2} \lambda^{4}}{4 \pi\left(m_{Q}^{2}+m_{\chi}^{2}\right)^{2}} \\
b \stackrel{r \rightarrow 0}{\simeq}-r \frac{m_{\chi}^{4}\left(2 m_{Q}^{2}+m_{\chi}^{2}\right) \lambda^{4}}{2 \pi\left(m_{Q}^{2}+m_{\chi}^{2}\right)^{4}}
\end{gathered}
$$

\section{B.3.2 Direct Detection Cross Section}

The effective Lagrangian for SI scattering for this model is

$$
\mathcal{L}_{\text {eff }}=\sum_{q}\left(f_{q} m_{q} \chi^{2} \bar{q} q+\frac{g_{q}^{(1)}}{m_{\chi}^{2}}\left(\chi \partial^{\mu} \partial^{\nu} \chi\right) \mathcal{O}_{\mu \nu}^{q}\right)
$$


and

$$
f_{q}=\frac{\lambda^{2}}{2\left(m_{Q}^{2}-m_{\chi}^{2}\right)}, \quad g_{q}^{(1)}=\frac{\lambda^{2} m_{\chi}^{2}}{\left(m_{Q}^{2}-m_{\chi}^{2}\right)^{2}} .
$$

In this Lagrangian, we have ignored terms with a $\gamma_{5}$ which are suppressed for nonrelativistic scattering. We use the relationship between scalar and fermion matrix elements

$$
\frac{\left\langle\chi\left|\chi^{2}\right| \chi\right\rangle_{\text {real scalar }}}{\langle\chi|\bar{\chi} \chi| \chi\rangle_{\text {Majorana fermion }}}=\frac{1}{2 m_{\chi}}
$$

where the denominator matrix element is twice as large compared to Dirac fermions, due to Majorana fermions being their own antiparticle. Thus, we can determine the spin-independent (SI) cross section by rescaling the Majorana case:

$$
\sigma_{\chi N}=\frac{\mu_{N}^{2}}{\pi}\left(\frac{f_{N}}{m_{\chi}}\right)^{2}
$$

where

$$
\frac{f_{N}}{m_{p}}=\sum_{q=u, d, s} f_{q} f_{T q}+\sum_{q=u, d, s, c, b} \frac{3}{4}(q(2)+\bar{q}(2)) g_{q}^{(1)}+\sum_{q=c, b, t} \frac{2}{27} c_{q} f_{q} f_{G}
$$

The second sum is the contribution from the twist-two operator and the third sum is the contribution from the heavy quarks $(c, b, t)$ to the nucleon mass [50] and contains the QCD correction factor $c_{q}$. Since we have not calculated the loop corrections to the gluons, we are unable to write down the short distance contributions to the scattering as in Eq. B.14.

\section{B.4 Complex Scalar Dark Matter}

\section{B.4.1 Relic Density}

$$
\begin{gathered}
a=\frac{3 \lambda^{4} m_{\chi}^{2}(1-r)^{3 / 2} r}{16 \pi\left(m_{Q}^{2}-m_{\chi}^{2}(r-1)\right)^{2}} \\
b=\frac{\lambda^{4} m_{\chi}^{2} \sqrt{1-r}}{128 \pi\left(m_{Q}^{2}-m_{\chi}^{2}(r-1)\right)^{4}}\left[m_{\chi}^{4}(r-1)^{2}\left(9 r^{2}-18 r+8\right)\right. \\
\left.-2 m_{Q}^{2} m_{\chi}^{2}\left(9 r^{3}-31 r^{2}+30 r-8\right)+m_{Q}^{4}\left(9 r^{2}-2 r+8\right)\right]
\end{gathered}
$$

To lowest order in $r$ we get,

$$
a \stackrel{r \rightarrow 0}{\simeq} \frac{3 \lambda^{4} m_{\chi}^{2} r}{16 \pi\left(m_{Q}^{2}+m_{\chi}^{2}\right)^{2}}
$$




$$
b \stackrel{r \rightarrow 0}{\simeq} \frac{\lambda^{4} m_{\chi}^{2}}{16 \pi\left(m_{Q}^{2}+m_{\chi}^{2}\right)^{2}}
$$

exhibiting the chiral suppression of $a$.

The effective Lagrangian following [49] is

$$
\mathcal{L}_{\mathrm{eff}}=\frac{i \lambda^{2}}{2\left(m_{Q}^{2}-m_{\chi}^{2}\right)} \bar{q} \gamma^{\mu} q \chi^{*} \partial_{\mu} \chi
$$

where we have ignored terms suppressed in the non relativistic limit. For the complex scalar we can relate its direct detection scattering rate to the fermionic case via

$$
\frac{\left\langle\chi\left|i \chi^{*} \partial^{\mu} \chi\right| \chi\right\rangle_{\text {scalar }}}{\left\langle\chi\left|\bar{\chi} \gamma^{\mu} \chi\right| \chi\right\rangle_{\text {fermion }}}=\frac{m_{\chi}}{2 m_{\chi}} \delta_{0}^{\mu}=\frac{1}{2} \delta_{0}^{\mu}
$$

Rescaling from the Dirac dark matter cross section in Eq. (B.20), we find a complex scalar cross section

$$
\sigma_{\mathrm{SI}}=\frac{9 \mu_{N}^{2} \lambda^{4}}{16 \pi\left(m_{Q}^{2}-m_{\chi}^{2}\right)^{2}}
$$

\section{B.5 Real Vector Dark Matter}

\section{B.5.1 Relic Density}

$$
\begin{gathered}
a=\frac{\lambda^{4} m_{\chi}^{2} \sqrt{1-r}\left(r^{2}-9 r+8\right)}{12 \pi\left(m_{Q}^{2}-m_{\chi}^{2}(r-1)\right)^{2}} \\
b=-\frac{\lambda^{4} m_{\chi}^{2} \sqrt{1-r}}{288 \pi\left(m_{Q}^{2}-m_{\chi}^{2}(r-1)\right)^{4}}\left[m_{\chi}^{4}(r-1)^{2}\left(17 r^{2}-92 r+112\right)\right. \\
\left.-2 m_{Q}^{2} m_{\chi}^{2}\left(17 r^{3}-69 r^{2}+132 r-80\right)+m_{Q}^{4}\left(17 r^{2}-12 r-80\right)\right]
\end{gathered}
$$

In the limit $r \rightarrow 0$,

$$
\begin{gathered}
a \stackrel{r \rightarrow 0}{\simeq} \frac{2 m_{\chi}^{2} \lambda^{4}}{3 \pi\left(m_{Q}^{2}+m_{\chi}^{2}\right)^{2}} \\
b \stackrel{r \rightarrow 0}{\simeq}-\frac{m_{\chi}^{2}\left(-5 m_{Q}^{4}+10 m_{Q}^{2} m_{\chi}^{2}+7 m_{\chi}^{4}\right) \lambda^{4}}{18 \pi\left(m_{Q}^{2}+m_{\chi}^{2}\right)^{4}}
\end{gathered}
$$




\section{B.5.2 Direct Detection}

We get the following effective Lagrangian for spin-independent interactions 47

$$
\mathcal{L}_{q}^{\mathrm{eff}}=f_{q}^{m} m_{q} \chi^{\mu} \chi_{\mu} \bar{q} q+\frac{g_{q}}{m_{\chi}^{2}} \chi^{\rho} i \partial^{\mu} i \partial^{\nu} \chi_{\rho} \mathcal{O}_{\mu \nu}^{q}+f_{G} \chi^{\rho} \chi_{\rho} G^{a \mu \nu} G_{\mu \nu}^{a}
$$

where $\mathcal{O}_{\mu \nu}^{q}$ and $\mathcal{O}_{\mu \nu}^{g}$ are the twist-2 operators as in (B.8) and (B.7). The total spinindependent scattering cross section per nucleon is

$$
\sigma_{\chi N}=\frac{1}{\pi m_{\chi}^{2}} \mu_{N}^{2}\left|f_{N}\right|^{2}
$$

where

$$
\begin{aligned}
& \frac{f_{N}}{m_{p}}=\sum_{q=u, d, s} f_{q} f_{T q}+\sum_{q=u, d, s, c, b} \frac{3}{4}(q(2)+\bar{q}(2)) g_{q}-\frac{8 \pi}{9 \alpha_{s}} f_{T G} f_{G}, \\
& f_{q}=-\frac{\lambda^{2} m_{Q}^{2}}{4\left(m_{Q}^{2}-m_{\chi}^{2}\right)^{2}}, \\
& g_{q}=-\frac{\lambda^{2} m_{\chi}^{2}}{\left(m_{Q}^{2}-m_{\chi}^{2}\right)^{2}}, \\
& f_{G} \simeq \frac{\alpha_{s} \lambda^{2}}{8 \pi}\left(\sum_{q=c, b, t} c_{q} \frac{m_{Q}^{2}}{6\left(m_{Q}^{2}-m_{\chi}^{2}\right)^{2}}+\sum_{q=a l l} \frac{1}{3\left(m_{Q}^{2}-m_{\chi}^{2}\right)}\right) .
\end{aligned}
$$

Here, we have taken for simplification the limiting values of $f_{G}$ for the small $m_{q}$ limit. Note that just like for the Majorana fermion model the first sum for $f_{G}$ is over the long distance contribution of the heavy quarks, where again there is a QCD correction factor $c_{q}$.

\section{B.6 Complex Vector Dark Matter}

\section{B.6.1 Relic Density}

$$
\begin{gathered}
a=\frac{\lambda^{4} m_{\chi}^{2} \sqrt{1-r}\left(-r^{2}-7 r+8\right)}{48 \pi\left(m_{Q}^{2}-m_{\chi}^{2}(r-1)\right)^{2}} \\
b=\frac{\lambda^{4} m_{\chi}^{2} \sqrt{1-r}}{1152 \pi\left(m_{Q}^{2}-m_{\chi}^{2}(r-1)\right)^{4}}\left[m_{\chi}^{4}(r-1)^{2}\left(25 r^{2}-74 r+40\right)\right. \\
\left.+2 m_{Q}^{2} m_{\chi}^{2}\left(-25 r^{3}+167 r^{2}-214 r+72\right)+m_{Q}^{4}\left(25 r^{2}-186 r+296\right)\right]
\end{gathered}
$$


In the limit $r \rightarrow 0$,

$$
\begin{gathered}
a \stackrel{r \rightarrow 0}{\simeq} \frac{m_{\chi}^{2} \lambda^{4}}{6 \pi\left(m_{Q}^{2}+m_{\chi}^{2}\right)^{2}} \\
b \stackrel{r \rightarrow 0}{\simeq} \frac{m_{\chi}^{2}\left(37 m_{Q}^{4}+18 m_{Q}^{2} m_{\chi}^{2}+5 m_{\chi}^{4}\right) \lambda^{4}}{144 \pi\left(m_{Q}^{2}+m_{\chi}^{2}\right)^{4}}
\end{gathered}
$$

\section{B.6.2 Direct Detection}

The dominant vector-vector spin independent interaction is

$$
\mathcal{L}_{\mathrm{eff}}=-\frac{i \lambda^{2}}{2\left(m_{Q}^{2}-m_{\chi}^{2}\right)} \bar{q} \gamma^{\mu} q \chi_{\nu}^{\dagger} \partial_{\mu} \chi^{\nu}
$$

To relate its direct detection scattering rate to the fermionic case we use

$$
\frac{\left\langle\chi\left|i \chi_{\nu}^{\dagger} \partial^{\mu} \chi^{\nu}\right| \chi\right\rangle_{\text {vector }}}{\left\langle\chi\left|\bar{\chi} \gamma^{\mu} \chi\right| \chi\right\rangle_{\text {fermion }}}=\frac{m_{\chi}}{2 m_{\chi}} \delta_{0}^{\mu}=\frac{1}{2} \delta_{0}^{\mu} .
$$

Rescaling from the Dirac dark matter cross section in Eq. B.20, we find a complex vector cross section

$$
\sigma_{\mathrm{SI}}=\frac{9 \mu_{N}^{2} \lambda^{4}}{16 \pi\left(m_{Q}^{2}-m_{\chi}^{2}\right)^{2}} .
$$

\section{References}

[1] M. Beltran, D. Hooper, E. W. Kolb, and Z. C. Krusberg, "Deducing the nature of dark matter from direct and indirect detection experiments in the absence of collider signatures of new physics," Phys.Rev. D80 (2009) 043509, arXiv:0808.3384 [hep-ph].

[2] J. Fan, M. Reece, and L.-T. Wang, "Non-relativistic effective theory of dark matter direct detection," JCAP 1011 (2010) 042, arXiv: 1008.1591 [hep-ph].

[3] A. L. Fitzpatrick, W. Haxton, E. Katz, N. Lubbers, and Y. Xu, "The Effective Field Theory of Dark Matter Direct Detection," JCAP 1302 (2013) 004, arXiv:1203.3542 [hep-ph].

[4] M. Beltran, D. Hooper, E. W. Kolb, Z. A. Krusberg, and T. M. Tait, "Maverick dark matter at colliders," JHEP 1009 (2010) 037, arXiv:1002.4137 [hep-ph]. 
[5] J. Goodman, M. Ibe, A. Rajaraman, W. Shepherd, T. M. Tait, et al., "Constraints on Light Majorana dark Matter from Colliders," Phys.Lett. B695 (2011) 185-188, arXiv:1005.1286 [hep-ph].

[6] Y. Bai, P. J. Fox, and R. Harnik, "The Tevatron at the Frontier of Dark Matter Direct Detection," JHEP 1012 (2010) 048, arXiv: 1005.3797 [hep-ph].

[7] J. Goodman, M. Ibe, A. Rajaraman, W. Shepherd, T. M. Tait, et al., "Constraints on Dark Matter from Colliders," Phys.Rev. D82 (2010) 116010, arXiv:1008.1783 [hep-ph].

[8] CDF Collaboration, T. Aaltonen et al., "A Search for dark matter in events with one jet and missing transverse energy in $p \bar{p}$ collisions at $\sqrt{s}=1.96 \mathrm{TeV}$," Phys.Rev.Lett. 108 (2012) 211804, arXiv:1203.0742 [hep-ex].

[9] "Search for new phenomena in monojet plus missing transverse momentum final states using $10 \mathrm{fb}-1$ of pp collisions at sqrts $=8$ tev with the atlas detector at the lhc," Tech. Rep. ATLAS-CONF-2012-147, CERN, Geneva, Nov, 2012.

[10] "Search for new physics in monojet events in pp collisions at sqrt(s)=8 tev," Tech. Rep. CMS-PAS-EXO-12-048, CERN, Geneva, 2013.

[11] A. Birkedal, K. Matchev, and M. Perelstein, "Dark matter at colliders: A Model independent approach," Phys.Rev. D70 (2004) 077701, arXiv:hep-ph/0403004 [hep-ph].

[12] J. L. Feng, S. Su, and F. Takayama, "Lower limit on dark matter production at the large hadron collider," Phys.Rev.Lett. 96 (2006) 151802, arXiv:hep-ph/0503117 [hep-ph].

[13] Q.-H. Cao, C.-R. Chen, C. S. Li, and H. Zhang, "Effective Dark Matter Model: Relic density, CDMS II, Fermi LAT and LHC," JHEP 1108 (2011) 018, arXiv:0912.4511 [hep-ph].

[14] K. Cheung, P.-Y. Tseng, Y.-L. S. Tsai, and T.-C. Yuan, "Global Constraints on Effective Dark Matter Interactions: Relic Density, Direct Detection, Indirect Detection, and Collider," JCAP 1205 (2012) 001, arXiv:1201.3402 [hep-ph].

[15] H. Dreiner, D. Schmeier, and J. Tattersall, "Contact Interactions Probe Effective Dark Matter Models at the LHC," arXiv:1303.3348 [hep-ph].

[16] M. Cirelli, N. Fornengo, and A. Strumia, "Minimal dark matter," Nucl.Phys. B753 (2006) 178-194, arXiv: hep-ph/0512090 [hep-ph]. 
[17] V. Silveira and A. Zee, "Scalar phantoms," Phys.Lett. B161 (1985) 136.

[18] J. McDonald, "Gauge singlet scalars as cold dark matter," Phys.Rev. D50 (1994) 3637-3649, arXiv:hep-ph/0702143 [HEP-PH].

[19] C. Burgess, M. Pospelov, and T. ter Veldhuis, "The Minimal model of nonbaryonic dark matter: A Singlet scalar," Nucl.Phys. B619 (2001) 709-728, arXiv:hep-ph/0011335 [hep-ph].

[20] J. M. Cline, K. Kainulainen, P. Scott, and C. Weniger, "Update on scalar singlet dark matter," arXiv:1306.4710 [hep-ph].

[21] G. Servant and T. M. Tait, "Is the lightest Kaluza-Klein particle a viable dark matter candidate?," Nucl.Phys. B650 (2003) 391-419, arXiv:hep-ph/0206071 [hep-ph].

[22] H.-C. Cheng, J. L. Feng, and K. T. Matchev, "Kaluza-Klein dark matter," Phys.Rev.Lett. 89 (2002) 211301, arXiv:hep-ph/0207125 [hep-ph].

[23] LHC New Physics Working Group Collaboration, D. Alves et al., "Simplified Models for LHC New Physics Searches," J.Phys. G39 (2012) 105005, arXiv:1105.2838 [hep-ph].

[24] B. Patt and F. Wilczek, "Higgs-field portal into hidden sectors," arXiv:hep-ph/0605188 [hep-ph].

[25] A. Djouadi, A. Falkowski, Y. Mambrini, and J. Quevillon, "Direct Detection of Higgs-Portal Dark Matter at the LHC," Eur.Phys.J. C73 (2013) 2455, arXiv:1205.3169 [hep-ph].

[26] M. Garny, A. Ibarra, M. Pato, and S. Vogl, "Closing in on mass-degenerate dark matter scenarios with antiprotons and direct detection," JCAP 1211 (2012) 017, arXiv:1207.1431 [hep-ph].

[27] Planck Collaboration, P. Ade et al., "Planck 2013 results. XVI. Cosmological parameters," arXiv:1303.5076 [astro-ph.CO].

[28] R. S. Chivukula and H. Georgi, "Composite Technicolor Standard Model," Phys.Lett. B188 (1987) 99.

[29] G. Jungman, M. Kamionkowski, and K. Griest, "Supersymmetric dark matter," Phys.Rept. 267 (1996) 195-373, arXiv:hep-ph/9506380 [hep-ph]. 
[30] XENON100 Collaboration, E. Aprile et al., "Dark Matter Results from 225 Live Days of XENON100 Data," Phys.Rev.Lett. 109 (2012) 181301. arXiv:1207.5988 [astro-ph.CO].

[31] DMtools. http://dmtools.brown.edu/.

[32] N. F. Bell, J. B. Dent, T. D. Jacques, and T. J. Weiler, "Electroweak Bremsstrahlung in Dark Matter Annihilation," Phys.Rev. D78 (2008) 083540 arXiv:0805.3423 [hep-ph].

[33] H. K. Dreiner, H. E. Haber, and S. P. Martin, "Two-component spinor techniques and Feynman rules for quantum field theory and supersymmetry," Phys.Rept. 494 (2010) 1-196, arXiv:0812.1594 [hep-ph].

[34] A. Geringer-Sameth and S. M. Koushiappas, "Exclusion of canonical WIMPs by the joint analysis of Milky Way dwarfs with Fermi," Phys.Rev.Lett. 107 (2011) 241303, arXiv:1108.2914 [astro-ph.C0].

[35] Fermi-LAT collaboration Collaboration, M. Ackermann et al., "Constraining Dark Matter Models from a Combined Analysis of Milky Way Satellites with the Fermi Large Area Telescope," Phys.Rev.Lett. 107 (2011) 241302, arXiv:1108.3546 [astro-ph.HE].

[36] CMS Collaboration, S. Chatrchyan et al., "Search for supersymmetry in hadronic final states with missing transverse energy using the variables $\alpha_{T}$ and b-quark multiplicity in pp collisions at $\sqrt{s}=8 \mathrm{TeV}$," $\operatorname{arXiv:1303.2985}$ [hep-ex].

[37] "Search for new physics in the multijets and missing momentum final state in proton-proton collisions at 8 tev," Tech. Rep. CMS-PAS-SUS-13-012, CERN, Geneva, 2013.

[38] "Search for top-squark pair production in the single lepton final state in pp collisions at 8 tev," Tech. Rep. CMS-PAS-SUS-13-011, CERN, Geneva, 2013.

[39] J. Alwall, M. Herquet, F. Maltoni, O. Mattelaer, and T. Stelzer, "MadGraph 5 : Going Beyond," JHEP 1106 (2011) 128, arXiv:1106.0522 [hep-ph].

[40] N. D. Christensen and C. Duhr, "FeynRules - Feynman rules made easy," Comput.Phys.Commun. 180 (2009) 1614-1641, arXiv:0806.4194 [hep-ph]. 
[41] J. Hisano, K. Ishiwata, and N. Nagata, "Direct Detection of Dark Matter Degenerate with Colored Particles in Mass," Phys.Lett. B706 (2011) 208-212, arXiv:1110.3719 [hep-ph].

[42] M. Gorchtein, S. Profumo, and L. Ubaldi, "Probing Dark Matter with AGN Jets," Phys.Rev. D82 (2010) 083514, arXiv:1008.2230 [astro-ph.HE].

[43] S. Chang, Y. Gao, and M. Spannowsky, "Enhanced Gamma Ray Signals in Cosmic Proton-Wimp Collisions Due to Hadronization," JCAP 1211 (2012) 053, arXiv: 1210.1870 [astro-ph.HE].

[44] K. Griest and D. Seckel, "Three exceptions in the calculation of relic abundances," Phys.Rev. D43 (1991) 3191-3203.

[45] M. Cahill-Rowley, R. Cotta, A. Drlica-Wagner, S. Funk, J. Hewett, et al., "Complementarity and Searches for Dark Matter in the pMSSM," arXiv:1305.6921 [hep-ph]

[46] E. W. Kolb and M. S. Turner, "The Early universe," Front.Phys. 69 (1990) $1-547$.

[47] J. Hisano, K. Ishiwata, N. Nagata, and M. Yamanaka, "Direct Detection of Vector Dark Matter," Prog.Theor.Phys. 126 (2011) 435-456, arXiv:1012.5455 [hep-ph].

[48] A. Djouadi and M. Drees, "QCD corrections to neutralino nucleon scattering," Phys.Lett. B484 (2000) 183-191, arXiv:hep-ph/0004205 [hep-ph].

[49] P. Agrawal, Z. Chacko, C. Kilic, and R. K. Mishra, "A Classification of Dark Matter Candidates with Primarily Spin-Dependent Interactions with Matter," arXiv:1003.1912 [hep-ph].

[50] M. A. Shifman, A. Vainshtein, and V. I. Zakharov, "Remarks on Higgs Boson Interactions with Nucleons," Phys.Lett. B78 (1978) 443. 